\title{
THE POROUS MEDIUM EQUATION IN ONE DIMENSION
}

BY

BARRY F. KNERR

\begin{abstract}
We consider a second order nonlinear degenerate parabolic partial differential equation known as the porous medium equation, restricting our attention to the case of one space variable and to the Cauchy problem where the initial data are nonnegative and have compact support consisting of a bounded interval. Solutions are known to have compact support for each fixed time.

In this paper we study the lateral boundary, called the interface, of the support $P[u]$ of the solution in $R^{1} \times(0, T)$. It is shown that the interface consists of two monotone Lipschitz curves which satisfy a specified differential equation. We then prove results concerning the behavior of the interface curves as $t$ approaches zero and as $t$ approaches infinity, and prove that the interface curves are strictly monotone except possibly near $t=0$. We conclude by proving some facts about the behavior of the solution in $P[u]$.
\end{abstract}

Introduction. Consider the parabolic Cauchy problem

$$
\begin{gathered}
u_{t}=\partial^{2} \phi(u) / \partial x^{2} \text { in } R^{1} \times(0, T), \\
u(x, 0)=u_{0}(x) \text { in } R^{1},
\end{gathered}
$$

where $u_{0}(x)$ is a bounded continuous nonnegative function and $\phi\left(u_{0}\right) \in$ $C^{0,1}\left(R^{1}\right)$. The function $\phi$ belongs to a class $\Phi_{0}$ of smooth positive functions that are like a function $\phi(u)=u^{m}$ for some real constant $m>1$. In particular, we assume $\phi(0)=\phi^{\prime}(0)=0$ and that $\phi(u), \phi^{\prime}(u)$ and $\phi^{\prime \prime}(u)$ are positive when $u>0$. Also, $\int_{0}^{1} \phi^{\prime}(\xi) / \xi d \xi$ and $\sup _{0<\xi<1}\left(\xi \phi^{\prime \prime}(\xi) / \phi^{\prime}(\xi)\right)$ are assumed to be finite. Notice that $(0.1)$ degenerates when $u=0$. When $\phi(u)=u^{m}(m>1)$, equation $(0.1)$ governs the flow of a gas in a porous medium where $u(x, t)$ is proportional to the denisty of the gas at $(x, t)$.

Several authors have studied $(0.1),(0.2)$ and other boundary value problems of (0.1). Oleinik, Kalashnikov and Yui-Lin [14] established the existence and uniqueness of a class of continuous weak solutions $u(x, t)$ of $(0.1),(0.2)$ and derived some elementary properties. They proved that $u$ is a classical solution of $(0.1)$ in the open set $P[u]$ wherein $u>0$, and that $u\left(x_{0}, t\right)>0$ for

Received by the editors January 27, 1976.

AMS (MOS) subject classifications (1970). Primary 35K15, 35K55; Secondary 35B99.

Key words and phrases. Nonlinear degenerate parabolic second order equation, porous medium. 
$t>\tau>0$ if $u\left(x_{0}, \tau\right)>0$. Also, they proved that if $u^{1}(x, t)$ and $u^{2}(x, t)$ are both weak solutions of $(0.1)$ and if $u_{1}(x, 0) \leqslant u_{2}(x, 0)$ for all $x \in R^{1}$, then $u_{1}(x, t) \leqslant u_{2}(x, t)$ for all $(x, t) \in R^{1} \times[0, T]$.

Weak solutions of $(0.1),(0.2)$ are particularly interesting when the support of $u_{0}(x)$ is some bounded interval $I=\left(a_{1}, a_{2}\right) \subset R^{1}$. For general $\phi \in \Phi_{0}$ it is known [14] that $u(\cdot, t)$ has compact support for each $t \in[0, T]$. This, it is well known, is not true for the Cauchy problem of the heat equation $u_{t}=u_{x x}$, and Kalashnikov [8] shows that it need not be true for weak solutions of $(0.1),(0.2)$ if the condition $\int_{0}^{1} \phi^{\prime}(\xi) / \xi d \xi<\infty$ is violated.

Much more is known when $\phi(u)=u^{m}(m>1)$. Kalashnikov [9] proved that the domain of dependence, $P[u]=\left\{(x, t) \in R^{1} \times(0, T) \mid u(x, t)>0\right\}$, is a domain bounded laterally by two monotone continuous curves $x=\zeta_{i}(t)$ $(i=1,2)$ where $\zeta_{1}(t)>a_{1}$ and $\zeta_{2}(t) \backslash a_{2}$ as $t \searrow 0$. These curves are called the interface curves. The weak solution $u(x, t)$ is not smooth at the interface; in fact, for arbitrarily large $\tau>0$, points of discontinuity of $u_{x}(x, t)$ can always be found in $\{t>\tau\}$ when $\phi(u)=u^{m}, m \geqslant 2$ (see [9]). Also, $\lim _{t \rightarrow \infty}\left|\zeta_{i}(t)\right|=$ $\infty$ [9]. Aronson [1], [2], [3] investigated the function $v(x, t)=u^{m-1}(x, t)$ which represents the pressure. He proved $\left|v_{x}\right|$ is bounded in $P[u] \cap\{t \geqslant \tau\}$ for any $\tau>0$ and that the same result holds for $\tau=0$ if $v_{0}=u_{0}^{m-1}$ is Lipschitz. He also showed that the interface curves are Lipschitz continuous and that the equation

$$
v_{x}\left(\zeta_{i}(t), t\right)\left\{\zeta_{i}^{\prime}(t)+\frac{m}{m-1} v_{x}\left(\zeta_{i}(t), t\right)\right\}=0
$$

holds for a.e. $t>0$. On the basis of Darcy's law we expect the equation

$$
\zeta_{i}^{\prime}(t)+\frac{m}{m-1} v_{x}\left(\zeta_{i}(t), t\right)=0
$$

to hold, in some sense, at the interface.

In this paper we consider the case of general $\phi \in \Phi_{0}$ and establish results for $u_{0}$ with support consisting of some bounded interval $I$. In $\$ I$ relevant terms are defined, some explicit weak solutions are exhibited (due to Barenblatt [4], [5]) and a function $v(x, t)=\psi(u(x, t))$ is defined. This function $v$ is given by $m u^{m-1} /(m-1)$ when $\phi(u)=u^{m}(m>1)$, and thus $v$ may be regarded as a generalization of the pressure function $u^{m-1}$ studied by Aronson. In $\S I I$ an a priori estimate of $\left|v_{x}\right|$ is given and in $\S \S I I I$ and IV existence, uniqueness and elementary properties established in [14] are summarized for their use in later sections. $\S \mathrm{V}$ develops some properties of equipotential curves of $v, v_{x}$ and $\partial \phi(u) / \partial x$ that are very useful in $\S \S \mathrm{VI}-\mathrm{XI}$.

The main results of the paper are proved in §§VI-XI. In §VI we show that $P[u]$ is bounded by monotone Lipschitz curves just as in the case $\phi(u)=u^{m}$ discussed above. Then in $\S$ VII we prove that the right derivative $\zeta_{i}^{\prime}(t)$ exists for all $t>0(i=1,2)$ and that 


$$
\zeta_{i}^{\prime}(t)=-v_{x}\left(\zeta_{i}(t), t\right) \text { for } t \in[0, T]
$$

(which is new even when $\phi(u)=u^{m}$ ). The initial growth of the interface is studied in §VIII. Here it is shown that a sufficient and almost necessary condition for an initially vertical interface is that $v(x, 0)$ be dominated by a parabola near the interface in question. Additionally it is shown that the interface curves grow like $t^{1 / 2}$ when the initial datum is the characteristic function $\chi_{I}(x)$. This is the fastest possible initial growth.

In §IX we show that the interface grows like $t^{1 /(m+1)}$ as $t \rightarrow \infty$ when $\phi(u)=u^{m}$. When $\phi(u) \in \Phi_{0}$ generally we show that the interface curves $x=\zeta_{i}(t)$ become infinite faster than $t^{1 /(m+1)}$ but slower than $t^{1 / 2}$ as $t \rightarrow \infty$, where $m>1$ is a constant depending on $\phi$.

In $\S \mathrm{X}$ we prove that the interface is either strictly monotone for all $t \geqslant 0$ or is initially vertical, for a finite period, and is thereafter strictly monotone. Instances of this latter case do indeed exist (see §VIII).

$\S \mathrm{XI}$ investigates the gradient $\left(v_{x}, v_{t}\right)$ of $v$ in $P[u]$. It is proved that the number of intervals on which $v_{x}(\cdot, t)$ is positive (negative) does not increase with time. We prove then that $v_{x}$ and $v_{t}$ do not vanish near the interface and that $v_{x}$ cannot vanish on a ball in $P[u]$.

I. Preliminaries. Let $G$ denote the domain $R^{1} \times(0, T)$ for some constant $T>0$ and let $\Phi_{0}$ denote the class of functions $\phi: R^{1} \rightarrow R^{1}$ satisfying:

$$
\begin{gathered}
\phi \in C^{1}[0, \infty) \cap C^{6+\alpha}(0, \infty), \\
\phi(0)=\phi^{\prime}(0)=0, \\
\phi(u)>0, \quad \phi^{\prime}(u)>0, \text { and } \phi^{\prime \prime}(u)>0 \text { if } u>0, \\
\int_{0}^{1} \frac{\phi^{\prime}(\xi)}{\xi} d \xi<\infty, \\
0<\sup _{0<\xi<1} \frac{\xi \phi^{\prime \prime}(\xi)}{\phi^{\prime}(\xi)}<\infty .
\end{gathered}
$$

It is easy to verify that for any constant $m>1$ the function $\phi(\xi)=\xi^{m}$ belongs to $\Phi_{0}$.

We shall consider the Cauchy problem

$$
\begin{gathered}
\partial u / \partial t=\partial^{2} \phi(u) / \partial x^{2} \text { in } G, \\
u(x, 0)=u_{0}(x) \text { in } R^{1},
\end{gathered}
$$

where $\phi \in \Phi_{0}$ and the function $u_{0}$ satisfies

$$
\phi\left(u_{0}\right) \in C^{0,1}\left(R^{1}\right), u_{0}>0 \text { on } I, \quad u_{0} \equiv 0 \text { on } R^{1} / I
$$

for some bounded interval $I=\left(a_{1}, a_{2}\right)$.

Some of the results derived in this paper are still valid if the condition 
$\phi \in \Phi_{0}$ is relaxed somewhat. For simplicity we shall, however, always assume $\phi \in \Phi_{0}$.

Although it is known that classical solutions of the Cauchy problem (1.6), (1.7) do not always exist [9], the existence and uniqueness of a class of weak solutions of the Cauchy problem, and other boundary value problems, was proved by Oleinik, Kalashnikov, and Yui-Lin [14], and an account of their treatment of the Cauchy problem, in English, may be found in [13]. When $u_{0} \in C\left(R^{1}\right)$ is a nonnegative bounded function, a weak solution of the Cauchy problem (1.6), (1.7) is defined to be a function $u \in C(\bar{G})$ having a weak derivative $\partial \phi(u) / \partial x$ and satisfying the integral identity

$$
\iint_{G}\left[\frac{\partial f}{\partial t} u-\frac{\partial f}{\partial x} \frac{\partial}{\partial x} \phi(u)\right] d x d t+\int_{-\infty}^{\infty} f(x, 0) u_{0}(x) d x=0
$$

for any function $f(x, t) \in C^{1}(\bar{G})$ which vanishes for large $|x|$ and for $t=T$. Uniqueness is proved directly from the definition by judicious choice of the function $f$ in (1.9).

Another problem considered in [14] is that of finding a solution $u$ of

$$
\begin{aligned}
\partial u / \partial t & =\partial^{2} \phi(u) / \partial x^{2} & & \text { in }(0, \infty) \times(0, T) \equiv H, \\
u(x, 0) & =u_{0}(x) & & \text { for } x \in[0, \infty), \\
u(0, t) & =u_{1}(t) & & \text { for } t \in[0, T],
\end{aligned}
$$

where $u_{0}$ and $u_{1}$ are nonnegative, bounded, continuous functions satisfying $u_{0}(0)=u_{1}(0)$. A bounded, continuous, nonnegative function $u(x, t)$ is said to be a weak solution of the boundary value problem (1.10)-(1.12) if $\phi(u)$ has a weak derivative $\partial \phi(u) / \partial x$ and if the following integral identity holds for every function $f \in C^{1}(\bar{H})$ which vanishes for $x=0, t=T$, and for large $x$ :

$$
\iint_{H}\left[\frac{\partial f}{\partial t} u-\frac{\partial f}{\partial x} \frac{\partial}{\partial x} \phi(u)\right] d x d t+\int_{0}^{\infty} f(x, 0) u_{0}(x) d x=0 .
$$

Uniqueness is proved by the same technique used in proving uniqueness for the Cauchy problem.

In the following sections it will often be advantageous to deal with a transformation $v=\psi(u)$ of a classical solution $u$ of (1.6) in some domain $D \subset G$ rather than the function $u$ itself. This transformation $\psi$ introduced by Oleinik, Kalashnikov and Yui-Lin [14] is given by

$$
\psi(s)=\int_{0}^{s} \frac{\phi^{\prime}(\xi)}{\xi} d \xi \text { for } s>0
$$

which is finite by virtue of (1.4). Thus we have

$$
v(x, t)=\psi(u(x, t))=\int_{0}^{u(x, t)} \frac{\phi^{\prime}(\xi)}{\xi} d \xi \text { in } D
$$


and

$$
\begin{cases}u_{t}=\frac{u}{\phi^{\prime}(u)} \frac{\partial}{\partial t} \psi(u)=\frac{u}{\phi^{\prime}(u)} v_{t} & \text { in } D, \\ (\phi(u))_{x}=\phi^{\prime}(u) u_{x}=u v_{x} & \text { in } D, \\ (\phi(u))_{x x}=u v_{x x}+u_{x} v_{x} & \text { in } D .\end{cases}
$$

It is easily verified that $v(x, t)$ satisfies the equation

$$
v_{t}=\sigma(v) v_{x x}+v_{x}^{2} \text { in } D
$$

where

$$
\sigma(s) \equiv \phi^{\prime}\left(\psi^{-1}(s)\right)=\left(\psi^{-1} /\left(\psi^{-1}\right)^{\prime}\right)(s) \text { for } s>0 .
$$

By direct computation we get $\sigma^{\prime}(s)=\xi \phi^{\prime \prime}(\xi) / \phi^{\prime}(\xi)$ where $\xi=\psi^{-1}(s)$, and if (1.5) holds then $0<\sigma^{\prime}<\infty$ on $(0,1)$.

In the physical case of gas filtration we deal with the special function

$$
\phi(u)=u^{m}, \quad m>1 .
$$

Then it is easily verified that in this case

$$
v=\psi(u)=m u^{m-1} /(m-1)
$$

and

$$
\sigma(v)=(m-1) v
$$

Consequently, (1.6) becomes

$$
u_{t}=\left(u^{m}\right)_{x x} \text { in } G
$$

and (1.17) becomes

$$
v_{t}=(m-1) v v_{x x}+v_{x}^{2} \text { in } G .
$$

It should be noted that Aronson [1], [2], [3] works with the function $v=$ $u^{m-1}$, which is not the same as the function $v$ in (1.20).

When $\phi(u)=u^{m}$, several explicit solutions are known due to Barenblatt [4], [5] (also see [15]). One weak solution of the Cauchy problem (1.22), (1.7) is given by

$$
u^{m-1}(x, t)=\lambda^{1-m}(t)\left(1-x^{2} \lambda^{-2}(t)\right)^{+},
$$

with initial data $u_{0}$ satisfying

$$
u_{0}(x, t)=C^{1-m}\left(1-x^{2} C^{-2}\right)^{+},
$$

where $C=(2 m(m+1) /(m-1))^{1 /(m+1)}$ and

$$
\lambda(t)=C(t+1)^{1 /(m+1)},
$$

and where $[x]^{+}=\max (x, 0)$. 
The function $u(x, t)$ given by

$$
u(x, t)=\left(A x^{2}\left(t_{2}-t\right)^{-1}\right)^{1 /(m-1)} \text { on } R^{1} \times\left(0, t_{2}\right),
$$

where $A=(m-1) /(2 m(m+1))$, is a classical solution of (1.22) with $T=$ $t_{2}$.

For general $\phi \in \Phi_{0}$ consider

$$
v(x, t)=C(C t-(x-a))^{+}
$$

where $C>0$ and $a \in R^{1}$ are constants. As in [14, Theorem 21] it is not difficult to see that the function $u(x, t)$ given by $u=\psi^{-1}(v)$, where $v$ is given by (1.28), is a weak solution of the boundary value problem (1.10)-(1.12) with the obvious boundary data $u_{0}(x)$ and $u_{1}(t)$.

These explicit solutions will be used later together with comparison theorems to derive various properties of weak solutions of the Cauchy problem (-1.6), (1.7). One reason for invoking the transformation $v=\psi(u)$ given by (1.15) is that the explicit solutions displayed above are of a simpler form when transformed and this resulting simplicity can be exploited with the aid of the maximum principle (see §IX).

S. Kamenomostskaya [10] introduced the transformation $u^{\prime}=c u, x^{\prime}=$ $x / L, t^{\prime}=c^{1-m} t / L^{2}$ for arbitrary positive real constants $c$ and $L$, under which equation (1.22) is invariant. Hence, if $u$ is a weak solution of (1.22), (1.7) it follows that

$$
\bar{u}(x, t)=c u\left(L x, c^{m-1} L^{2} t\right)
$$

is also a weak solution of (1.22), (1.7) with

$$
\bar{u}_{0}(x)=c u(L x, 0) \quad \text { when } x \in R^{1} .
$$

Also, if $u(x, t)$ is a weak solution of (1.22), (1.7) and if we define

$$
\bar{v}(x, t) \equiv k v\left(L x, k L^{2} t\right)
$$

where $v=\psi(u)=(m /(m-1)) u^{m-1}$, then it is easy to see that the function $\bar{u}(x, t)$ given by

$$
\bar{u}(x, t) \equiv \psi^{-1} \bar{v}(x, t)
$$

is also a weak solution of $(1.22)$ and $\bar{u}(x, 0)=\psi^{-1}(k v(L x, 0))$.

II. An a priori estimate of $\sup \left|v_{x}\right|$.

LEMma 2.1. Let $\phi \in \Phi_{0}$ and let $R$ denote the rectangle $(a, b) \times(0, T]$. Suppose $v \in \bar{C}_{2+\alpha}(R), v_{x x x} \in C(R), 0<v<N$, in $R$ and $v$ is a classical solution of the parabolic equation

$$
v_{t}=\sigma(v) v_{x x}+v_{x}^{2}
$$

in $R$, where $\sigma$ is defined in (1.18). Let $R^{*}=\left(a_{1}, b_{1}\right) \times(\tau, T]$ be a rectangle 
with $a<a_{1}, b_{1}<b$ and $0<\tau<T$, a subrectangle of $R$. Then

$$
\left|v_{x}(x, t)\right| \leqslant C \text { in } \bar{R}^{*}
$$

where $C$ depends only on $a_{1}-a, b-b_{1}, k$, and $\tau$, where

$$
k=\sup _{0<s<N} \sigma^{\prime}(s)
$$

(recall $0<k<\infty$ by (1.5) and (1.18)). If $M_{1}=\sup _{(a, b)}\left|v_{x}(x, 0)\right|<\infty$, then the same conclusion holds for $R^{*}=\left(a_{1}, b_{1}\right) \times(0, T]$ where $C$ now depends on $M_{1}$ instead of $\tau$.

Aronson [3] proved this very useful result in the special case $\phi(u)=u^{m}$. His proof, with no modification, also proves Lemma 2.1. A detailed sketch of the proof can also be found in [7].

III. Existence theorems. The existence of weak solutions of both the Cauchy problem (1.6), (1.7) and the problem (1.10)-(1.12) was proved in [14]. For future reference we shall now state these results and some variants.

THEOREM 3.1. Let $\phi \in \Phi_{0}$ and suppose $u_{0}(x)$ to be a continuous, bounded, and nonnegative function and let $\phi\left(u_{0}\right)$ be a Lipschitz continuous function. Then there exists a unique weak solution $u$ of (1.6), (1.7) which is a classical solution of (1.2) in the open set $\{u>0\}$.

THEOREM 3.2. Let $\phi \in \Phi_{0}$ and suppose $u_{0}(x)$ and $u_{1}(t)$ are bounded, nonnegative and continuous functions on $[0, \infty)$ and $[0, T]$, respectively, such that $u_{0}(0)=u_{1}(0)$. Also suppose $\psi\left(u_{0}\right) \in C^{0,1}[0, \infty)$ and $\psi\left(u_{1}\right) \in C^{0,1}[0, T]$. Then there exists a unique weak solution $u(x, t)$ of the problem (1.10)-(1.12) and $u(x, t)$ is a classical solution of $(1.10)$ in the open set $\{u>0\}$.

REMARK 3.3. If, in the hypotheses of Theorem 3.2, we require only $\phi\left(u_{0}\right) \in$ $C^{0,1}[0, \infty)$ and $\phi\left(u_{1}\right) \in C^{0,1}[0, T]$ instead of $\psi\left(u_{0}\right) \in C^{0,1}$ and $\psi\left(u_{1}\right) \in C^{0,1}$, and if we require of $\phi$ that it satisfy $\sup _{\xi>0}\left\{\left(\phi^{\prime}(\xi)\right)^{2} / \phi^{\prime \prime}(\xi)\right\}<\infty$, instead of (1.5), then the conclusions of the theorem are still valid (see [14]).

The proofs of these theorems given in [14] produce the weak solution $u$ as $u=\Phi(w)$ where $\Phi=\phi^{-1}$ and where $w$ is the pointwise limit of a monotone decreasing sequence of smooth positive functions $w_{n}(x, t)$ which solve some initial-boundary value problem of the equation

$$
w_{x x}=\Phi^{\prime}(w) w_{t}
$$

in the rectangle $G_{n} \equiv(-n, n) \times(0, T)$. The initial data $w_{0 n}(x)$, and $\partial w_{0 n}(x) / \partial x$ are uniformly bounded in $n$, and the lateral data are just $\sup _{x} w_{0 n}(x)$. We mention this here because later on we shall make use of these facts. 
IV. Elementary properties of weak solutions. Theorems $4.1-4.3$ below were proved in [14]. We recall them now for future reference.

TheOREM 4.1. Let $\phi \in \Phi_{0}$ and suppose $u_{1}(x, t)$ and $u_{2}(x, t)$ are weak solutions of the Cauchy problem (1.6), (1.7) with initial data $u_{0}^{1}(x)$ and $u_{0}^{2}(x)$ such that both $\phi\left(u_{0}^{1}(x)\right)$ and $\phi\left(u_{0}^{2}(x)\right)$ are Lipschitz continuous. If

$$
u_{0}^{1}(x) \leqslant u_{0}^{2}(x) \quad \text { for } x \in R^{1}
$$

then

$$
u_{1}(x, t) \leqslant u_{2}(x, t) \quad \text { for }(x, t) \in G .
$$

THEOREM 4.2. Let $\phi \in \Phi_{0}$ and suppose $u_{1}(x, t)$ and $u_{2}(x, t)$ are weak solutions of (1.10) with data $u_{i}(x, 0)=u_{0}^{i}(x)$ for $x \geqslant 0(i=1,2)$ and $u_{i}(0, t)=$ $u_{1}^{i}(t)$ for $0 \leqslant t \leqslant T(i=1,2)$ such that $\psi\left(u_{0}^{i}\right)$ and $\psi\left(u_{1}^{i}\right)(i=1,2)$ are Lipschitz continuous. If $u_{k}^{1} \leqslant u_{k}^{2}(k=0,1)$ then

$$
u_{1}(x, t) \leqslant u_{2}(x, t) \quad \text { in } \bar{H}=[0, \infty) \times[0, T] .
$$

In [14], Theorem 4.2 was proved with slightly different conditions on $\phi$ and the initial data (see Remark 3.3).

THEOREM 4.3. Suppose $\phi \in \Phi_{0}$ and $u(x, t)$ is a weak solution of (1.6), (1.7.) or (1.10)-(1.12). If $u\left(x_{0}, t_{0}\right)>0$ where $\left(x_{0}, t_{0}\right) \in G$, then $u\left(x_{0}, t\right)>0$ for $t>t_{0}$.

THEOREM 4.4. Suppose $\phi \in \Phi_{0}$ and $u(x, t)$ is a weak solution of the Cauchy problem (1.6), (1.7) where $\phi\left(u_{0}\right)$ is Lipschitz continuous. Then, for the function $v(x, t)$ defined by $(1.15)$ we have

$$
\left|v(x, t)-v\left(x^{\prime}, t\right)\right| \leqslant C\left|x-x^{\prime}\right|
$$

for all $(x, t)$ and $\left(x^{\prime}, t\right)$ in $(-\infty, \infty) \times(\tau, T)$ where $C>0$ depends only on $\tau$ and $k=\sup \sigma^{\prime}$ on $(0, \sup v)$. If $v_{0}=\psi\left(u_{0}\right)$ is Lipschitz continuous and

$$
\left|v_{0}(x)-v_{0}\left(x^{\prime}\right)\right| \leqslant N_{1}\left|x-x^{\prime}\right| \text { for } x, x^{\prime} \in R,
$$

then the same conclusions hold with $\tau=0$ except that $C$ now depends on $N_{1}$ instead of $\tau$.

Proof. Recall that $\phi(u)$ is the pointwise limit of a monotone decreasing sequence of functions $w_{n} \in \bar{C}_{2+\alpha}\left(G_{n}\right)$ which are positive solutions of (3.1). Let $v_{n}=\psi \circ \Phi\left(w_{n}\right)$ and note that $v_{n} \in \bar{C}_{2+\alpha}\left(G_{n}\right)$ are solutions of (1.17) in $G_{n}$. By [6, Theorem 10, Chapter 3], it follows that $\left(v_{n}\right)_{x x x}$ is continuous in $G_{n}$.

Since the sequence $\left\{v_{n}\right\}$ is positive and monotone decreasing to $v$, it suffices to establish the validity of $(4.5)$ for each $v_{n}$ with a constant $C$ that does not depend on $n$. In the case of the first assertion this is obvious because each $v_{n}$ is a solution of (2.1) with a function $\sigma$ defined by (1.18) which clearly satisfies all of the conditions of Lemma 2.1.

To prove the second assertion we need only show that the sequence $\left\{w_{n}^{0}\right\}$ 
can be chosen in such a way that the functions $(\psi \circ \Phi)\left(w_{n}^{9}\right)$ are Lipschitz continuous with a Lipschitz constant that does not depend on $n$. To do this, first construct a sequence of positive monotone decreasing $C^{\infty}$ functions $\left\{g_{n}\right\}$ which converge uniformly on compact sets to $\psi\left(u_{0}\right)$ and whose derivatives $\left\{g_{n}^{\prime}\right\}$ are bounded uniformly in absolute value by a constant that depends only on $N_{1}$. Then the functions $w_{n}^{0}=\phi \circ \psi^{-1} g_{n}$ are positive and monotone decreasing to $\phi\left(u_{0}\right)$ and

$$
\left|d w_{0}^{n} / d x\right|=\left|\psi^{-1}\left(g_{n}\right)\right|\left|g_{n}^{\prime}\right|
$$

follows from (1.16). This completes the proof.

A similar theorem was proved by Aronson [3] in the case $\phi(u)=u^{m}$.

V. Equipotential curves. Throughout this section we assume that $\phi \in \Phi_{0}$ and $v(x, t)$ is a classical solution of $(2.1)$ in some domain $D \subset G$.

LEMMA 5.1. Let $\left(x_{0}, t_{0}\right) \in D, v_{x}\left(x_{0}, t_{0}\right)=\alpha$ and suppose

$$
v_{x x}\left(x_{0}, t_{0}\right)=0, \quad v_{x t}\left(x_{0}, t_{0}\right) \neq 0 .
$$

Then there exist a neighborhood

$$
N_{\delta}=\left(x_{0}-\delta, x_{0}+\delta\right) \times\left(t_{0}-\delta, t_{0}+\delta\right)
$$

in $D$ and a function $\gamma(x) \in C^{2}\left(x_{0}-\delta, x_{0}+\delta\right)$ such that $\left(v_{x}\right)^{-1}(\{\alpha\}) \cap N_{\delta}=$ $\left\{(x, \gamma(x))|| x-x_{0} \mid<\delta\right\}$. Furthermore $\gamma^{\prime}\left(x_{0}\right)=0$ and $\gamma^{\prime \prime}\left(x_{0}\right)<0$ (so that $\gamma$ takes a relative maximum at $\left.x_{0}\right)$. Finally the function $v_{x x}(x, \gamma(x))$ vanishes only at $x_{0}$ for $\left|x-x_{0}\right|<\delta$ and it has a different algebraic sign in the intervals $x_{0}-\delta<x<x_{0}$ and $x_{0}<x<x_{0}+\delta$.

Proof. By [6, Theorem 11, Chapter 3], it follows that since $\sigma \in C_{5+\alpha}(0, \infty)$ the derivatives $v_{x x x x}, v_{x x t}$ and $v_{x x t t}$ are in $C_{\alpha}(D)$. Let $\delta>0$ be small enough that $v_{x t}$ does not vanish in $N_{\delta}$ (then for fixed $x$ the mapping $t \rightarrow v_{x}(x, t)$ is one-to-one in $N_{\delta}$ ). By the implicit function theorem there exists a unique function $\gamma \in C^{2}\left(x_{0}-\delta, x_{0}+\delta\right)$ such that $\gamma\left(x_{0}\right)=t_{0}$ and

$$
v_{x}(x, \gamma(x))=\alpha \quad \text { for }\left|x-x_{0}\right|<\delta .
$$

Taking a derivative in (5.2) we get

$$
v_{x x}+v_{x t} \gamma^{\prime}=0 \text { when }\left|x-x_{0}\right|<\delta .
$$

But since $v_{x x}\left(x_{0}, t_{0}\right)=0$ and $v_{x t}\left(x_{0}, t_{0}\right) \neq 0$ we conclude that

$$
\gamma^{\prime}\left(x_{0}\right)=0 \text {. }
$$

Differentiating (5.3) yields, for $\left|x-x_{0}\right|<\delta$,

$$
v_{x x x}+v_{x x t} \gamma^{\prime}+v_{x t} \gamma^{\prime \prime}+\gamma^{\prime}\left(v_{x x t}+v_{x t l} \gamma^{\prime}\right)=0
$$

and we note that (5.4) and (5.5) imply

$$
v_{x x x}\left(x_{0}, t_{0}\right)+v_{x t}\left(x_{0}, t_{0}\right) \gamma^{\prime \prime}\left(x_{0}\right)=0 \text {. }
$$


Differentiating (2.1) with respect to $x$ yields

$$
v_{x t}=\sigma(v) v_{x x x}+\left(\sigma^{\prime}(v)+2\right) v_{x} v_{x x} \text { in } N_{\delta}
$$

and, since $v_{x x}\left(x_{0}, t_{0}\right)=0$,

$$
v_{x t}\left(x_{0}, t_{0}\right)=\sigma\left(v\left(x_{0}, t_{0}\right)\right) v_{x x x}\left(x_{0}, t_{0}\right) .
$$

Combining (5.6) and (5.8) we get

$$
v_{x x x}\left(x_{0}, t_{0}\right)+\sigma\left(v\left(x_{0}, t_{0}\right)\right) v_{x x x}\left(x_{0}, t_{0}\right) \gamma^{\prime \prime}\left(x_{0}\right)=0 \text {. }
$$

By (5.1) and (5.8) $v_{x x x}\left(x_{0}, t_{0}\right) \neq 0$ and, consequently,

$$
\gamma^{\prime \prime}\left(x_{0}\right)=-1 / \sigma\left(v\left(x_{0}, t_{0}\right)\right)<0 .
$$

Therefore $\gamma^{\prime}(x)>0$ for $x_{0}-\delta<x<x_{0}$ and $\gamma^{\prime}(x)<0$ for $x_{0}<x<x_{0}+\delta$ (with perhaps a smaller $\delta$ ). In view of (5.3) and the fact that $v_{x t} \neq 0$ in $N_{\delta}$, it is evident that $v_{x x}(x, \gamma(x))$ vanishes only at $x=x_{0}$ for $\left|x-x_{0}\right|<\delta$ and it has a different sign on the intervals $\left(x_{0}-\delta, x_{0}\right)$ and $\left(x_{0}, x_{0}+\delta\right)$.

By similar proofs we get the next two lemmas.

LEMMA 5.2. Let $\left(x_{0}, t_{0}\right) \in D$ and $v\left(x_{0}, t_{0}\right)=\varepsilon$ and suppose

$$
v_{x}\left(x_{0}, t_{0}\right)=0, \quad v_{t}\left(x_{0}, t_{0}\right) \neq 0 \text {. }
$$

Then there exist a neighborhood $N_{\delta}$ of $\left(x_{0}, t_{0}\right)$ in $D$ and a function

$$
\gamma(x) \in C^{2}\left(x_{0}-\delta, x_{0}+\delta\right)
$$

such that $v^{-1}(\{\varepsilon\}) \cap N_{\delta}$ is given by $\left\{(x, \gamma(x))|| x-x_{0} \mid<\delta\right\}$. Furthermore $\gamma^{\prime}\left(x_{0}\right)=0, \gamma^{\prime \prime}\left(x_{0}\right)<0$, the function $v_{x}(x, \gamma(x))$ vanishes only at $x_{0}$ for $\left|x-x_{0}\right|$ $<\delta$ and the sign of this function is different on the two intervals $\left(x_{0}-\delta, x_{0}\right)$ and $\left(x_{0}, x_{0}+\delta\right)$.

LEMMA 5.3. If $w$ is a positive classical solution of

$$
\Phi^{\prime}(w) w_{t}=w_{x x}
$$

in $D\left(\Phi=\phi^{-1}\right)$, then the conclusions of Lemma 5.1 hold with $v$ replaced by $w$.

LEMMA 5.4. Suppose all of the hypotheses of Lemma 5.2 are fulfilled. Let $D \subset G$ be a bounded open set such that $D^{\prime}=\operatorname{int}(\partial D \cap\{t=0\})$ is a nonempty open set in $R^{1}$. Also suppose $v \in C(\bar{D})$ and is positive on $D \cup D^{\prime}$. Let $\left(x_{0}, t_{0}\right) \in D, v\left(x_{0}, t_{0}\right)=\varepsilon$ where $\varepsilon$ is a noncritical value of $v\left(i . e .\left(v_{x}, v_{t}\right) \neq 0\right.$ on the set $\left.v^{-1}(\{\varepsilon\}) \cap D\right)$. Suppose $v=0$ on $\partial D \cap\left\{0<t<t_{0}\right\}$. Then there exists a level curve $x=\beta(t) \in C\left(0, t_{0}\right]$, lying in $D$, along which $v=\varepsilon$ and $v_{x}$ never vanishes, except perhaps at $t=t_{0}$. That is, $\beta\left(t_{0}\right)=x_{0}, v(\beta(t), t)=\varepsilon$ and $v_{x}(\beta(t), t) \neq 0$ for $t \in\left(0, t_{0}\right)$.

Proof. We shall first show that a level curve exists locally. Since $\varepsilon$ is noncritical, either $v_{x}\left(x_{0}, t_{0}\right) \neq 0$ or $v_{t}\left(x_{0}, t_{0}\right) \neq 0$. If $v_{x}\left(x_{0}, t_{0}\right) \neq 0$ then $v_{x} \neq 0$ in a neighborhood of $P_{0}=\left(x_{0}, t_{0}\right)$ and for fixed $t$ the map $x \rightarrow v(x, t)$ is 
one-to-one locally. This implies that there can be at most one curve through $P_{0}$ along which $v=\varepsilon$. The implicit function theorem guarantees the local existence of such a continuous curve. Without loss of generality $v_{x} \neq 0$ along this curve. If, on the other hand, $v_{x}\left(P_{0}\right)=0$ then, since $\varepsilon$ is a noncritical value, $v_{t}\left(P_{0}\right) \neq 0$ and Lemma 5.2 applies, furnishing a desired curve. In fact, Lemma 5.2 gives us the choice of two curves, one being the inverse function of $\gamma$ for $x<x_{0}$ and the other being the inverse of $\gamma$ for $x>x_{0}$. These inverse functions exist because locally $\gamma^{\prime}$ vanishes only at $x_{0}$. We have therefore shown that there exist a number $\delta_{0}>0$ and a function $\beta_{\delta_{0}}(t) \in C\left[t_{0}-\delta_{0}, t_{0}\right]$ such that $v(\beta(t), t)=\varepsilon$ for $t \in\left[t_{0}-\delta, t_{0}\right]$ and $v_{x}(\beta(t), t) \neq 0$ for $t \in\left[t_{0}-\right.$ $\left.\delta, t_{0}\right)$.

A function $\beta_{\delta}(t) \in C\left(t_{0}-\delta, t_{0}\right.$ ] for some positive constant $\delta>\delta_{0}$ will be called an extension of $\beta_{\delta_{0}}$ if we have:

(i) $\left(\beta_{\delta}(t), t\right) \in D \quad\left(t \in\left(t_{0}-\delta, t_{0}\right]\right)$,

(ii) $v_{x}\left(\beta_{\delta}(t), t\right) \neq 0 \quad\left(t \in\left(t_{0}-\delta, t_{0}\right)\right)$,

(iii) $v\left(\beta_{\delta}(t), t\right)=\varepsilon \quad\left(t \in\left(t_{0}-\delta, t_{0}\right)\right)$,

(iv) $\beta_{\delta}(t)=\beta_{\delta_{0}}(t) \quad\left(t \in\left(t_{0}-\delta_{0}, t_{0}\right]\right)$.

We shall prove there exists a unique maximal extension of $\beta_{\delta_{0}}$. First note that if $\beta_{\delta_{1}}$ and $\beta_{\delta_{2}}$ are extensions of $\beta_{\delta_{0}}$ and $\delta_{1}<\delta_{2}$, then $\beta_{\delta_{1}}(t)=\beta_{\delta_{2}}(t)$ for $t \in\left(t_{0}-\delta_{1}, t_{0}\right]$. Indeed, if $\bar{\delta}=\sup _{\delta>\delta_{0}}\left\{\delta \mid \beta_{\delta_{1}}\left(t_{0}-\delta\right)=\beta_{\delta_{2}}\left(t_{0}-\delta\right)\right\}$, then $\delta_{0}<\bar{\delta}<\delta_{1}$. If $\bar{\delta}=\delta_{1}$ the result is immediate, so assume $\bar{\delta}<\delta_{1}$. Then $\beta_{\delta_{1}}\left(t_{0}-\bar{\delta}\right)=\beta_{\delta_{2}}\left(t_{0}-\bar{\delta}\right)=\bar{x}$ and $v_{x}\left(\bar{x}, t_{0}-\bar{\delta}\right) \neq 0$. Then $v_{x}$ does not vanish in some ball $B$ centered at $\left(\bar{x}, t_{0}-\bar{\delta}\right)$ and, consequently, in $B, v$ is $1-1$ as a function of $x$ for each fixed $t$. However, this is impossible since there are values of $t$ smaller than and arbitrarily close to $t_{0}-\bar{\delta}$ such that $\beta_{\delta_{1}}(t) \neq$ $\beta_{\delta_{2}}(t)$ and $v\left(\beta_{\delta_{1}}(t), t\right)=v\left(\beta_{\delta_{2}}(t), t\right)=\varepsilon$.

Let $\delta^{*}$ be the supremum of the set of $\delta>\delta_{0}$ for which there exists an extension $\beta_{\delta}$ of $\beta_{\delta_{0}}$. Then $\delta_{0}<\delta^{*}<t_{0}$ and clearly there exists an extension $\beta_{\delta^{*}}$ of $\beta_{\delta_{0}}$, which is necessarily unique. If $\delta^{*}=t_{0}$ then $\beta_{\delta^{*}}$ is the sought curve. We shall therefore assume $\delta^{*}<t_{0}$ and derive a contradiction. Since $D$ is bounded, it follows that there exists a sequence of points $\left\{P_{n}=\left(\beta_{\delta^{*}}\left(t_{n}\right), t_{n}\right)\right\}$ such that $t_{n} \backslash t_{0}-\delta^{*}>0, t_{n} \neq t_{n+1}$, and $\beta_{\delta^{*}}\left(t_{n}\right) \rightarrow x^{*}$ where $P=\left(x^{*}, t_{0}-\right.$ $\left.\delta^{*}\right) \in \bar{D}$. Since, by hypothesis, $v$ vanishes on $\partial D \cap\left\{0<t<t_{0}\right\}$ and since $v=\varepsilon$ at $P$, it follows that $P \in D$ and $\nabla v(P) \neq 0$. If $v_{x}(P)=0$ then $v_{t}(P) \neq 0$ and by Lemma 5.2 there exists a neighborhood $N$ of $P$ such that $v^{-1}(\{\varepsilon\}) \cap N$ is a curve $t=\gamma(x)$ attaining a relative maximum at $x=\bar{x}$, which implies $v \neq \varepsilon$ in $N \cap\left\{t>t_{0}-\delta^{*}\right\}$. But for large $n$ each $P_{n}$ is in $N \cap\left\{t>t_{0}-\delta^{*}\right\}$; a contradiction. From this contradiction we conclude that $v_{x}(P) \neq 0$. But then the implicit function theorem implies that $\beta_{\delta^{*}}$ can be extended, which contradicts the maximality of $\beta_{\delta^{*}}$. This contradiction forces us to conclude $\delta^{*}=t_{0}$, which concludes the proof. 
REMARK 5.5. Note that $\beta$ is unique if $v_{x}\left(x_{0}, t_{0}\right) \neq 0$, but there are precisely two curves if $v_{x}\left(x_{0}, t_{0}\right)=0$.

REMARK 5.6. Except for at most a countable number of values $\varepsilon$, $\lim _{t>0} \beta(t)$ exists. Indeed, if $\lim _{t>0} \beta(t)$ does not exist then $v \equiv \varepsilon$ on some nonempty interval $B \subset D^{\prime}$, since there exist two sequences $\left\{P_{n}=\left(\beta\left(t_{n}\right), t_{n}\right)\right\}$ and $\left\{P_{n}^{\prime}=\left(\beta\left(t_{n}^{\prime}\right), t_{n}^{\prime}\right)\right\}$ such that $t_{n}$ and $t_{n}^{\prime}$ decrease strictly to zero and $t_{n}<t_{n}^{\prime}$ and such that $\beta\left(t_{n}\right) \rightarrow x$ and $\beta\left(t_{n}^{\prime}\right) \rightarrow x^{\prime}$ where $x<x^{\prime}$ and $x, x^{\prime} \in D^{\prime}$. But then, because of the continuity of $\beta$, for any point $x^{\prime \prime}$ in $\left(x, x^{\prime}\right)$ there must be a value $t_{n}^{\prime \prime}$ such that $\beta\left(t_{n}^{\prime \prime}\right)=x^{\prime \prime}$ and $t_{n}<t_{n}^{\prime \prime}<t_{n}^{\prime}$; hence, $v\left(x^{\prime \prime}, t_{n}^{\prime \prime}\right)=\varepsilon$. Then since $v \in C(\bar{D})$ it follows that $v\left(x^{\prime \prime}, 0\right)=\varepsilon$ for this arbitrary $x^{\prime \prime}$ in $\left(x, x^{\prime \prime}\right)$.

We conclude that $\beta \in C\left[0, t_{0}\right]$ if $\varepsilon$ is not in $S$ where $S$ is the collection of $\alpha$ in $R^{\prime}$ such that $v \equiv \alpha$ on some interval $I_{\alpha} \subset D^{\prime}$. But since $D^{\prime}$, being a bounded open set in $R^{1}$, can contain only a countable number of disjoint intervals $I_{\alpha}$ it follows that $S$ is countable.

LEMMA 5.7. Let $D \subset G$ be $a$ bounded open set and suppose $v(x, t)$ is $a$ classical solution of (2.1) in $D$. If $\alpha$ is a noncritical value of $v_{x}$ and if $v_{x}\left(x_{0}, t_{0}\right)=\alpha$ where $\left(x_{0}, t_{0}\right) \in D$ then there exists a constant $t^{*}, 0<t^{*} \leqslant t_{0}$, and a curve $X=\beta(t) \in C\left(t^{*}, t_{0}\right]$ lying in $D$, passing through $\left(x_{0}, t_{0}\right)$, along which $v_{x} \equiv \alpha$ and $v_{x x}$ does not vanish, except perhaps at $\left(x_{0}, t_{0}\right)$. Furthermore, if $t^{*}>0$ then $\lim _{t \rightarrow t^{*}} \beta(t)$ exists and $\left(\beta\left(t^{*}\right), t^{*}\right) \in \partial D$.

Proof. The proof is similar to the proof of Lemma 5.4 and employs Lemma 5.1. We omit the details.

\section{The domain of dependence $P[u]$.}

THEOREM 6.1. Let $u$ be a weak solution of (1.6), (1.7) with initial data $u_{0}$ satisfying (1.8) and let $P[u]=\{(x, t) \in G \mid u(x, t)>0\}$. Then there exists curves $x=\zeta_{1}(t)$ and $x=\zeta_{2}(t)$ in $C[0, T] \cap C^{0,1}[\tau, T]$ for any $\tau>0$ such that $\zeta_{i}(0)=a_{i}, \zeta_{1}>, \zeta_{2} \rightarrow$ and

$$
P[u]=\left\{(x, t) \mid \zeta_{1}(t)<x<\zeta_{2}(t), 0<t<T\right\} .
$$

Also, if $v_{0} \in C^{0,1}\left(R^{1}\right)$ then $\zeta_{i} \in C^{0,1}[0, T](i=1,2)$.

We shall first prove that for arbitrary $\tau \in(0, T)$ the set $P[u] \cap\{t=\tau\}$ is an open interval. By Theorem 4.3, $P[u] \cap\{t=\tau\} \neq \varnothing$. Let $a_{\tau}=\inf \{x \mid(x, \tau)$ $\in P[u]\}$ and $b_{\tau}=\sup \{x \mid(x, \tau) \in P[u]\}$. Theorem 21 [14] implies that $a_{\tau}$ and $b_{\tau}$ are finite and it follows from the continuity of $v$ that $v\left(a_{\tau}, \tau\right)=v\left(b_{\tau}, \tau\right)=$ 0 .

Let $C$ denote the set of critical values of $v$ together with the countable set of values $\left\{\varepsilon_{n}\right\}$ for which it is possible that $v \equiv \varepsilon_{n}$ on some interval contained in $I$ (see Remark 5.6). By Sard's theorem, $C$ has measure zero. We shall now show that there exist two sequences $\left\{a^{n}\right\}$ and $\left\{b^{n}\right\}$ such that the points 
$\left(a^{n}, \tau\right)$ and $\left(b^{n}, \tau\right)(n \geqslant 1)$ are in $P[u]$ and

(i) $a^{n} \searrow a_{\tau}$ and $b^{n} \rightarrow b_{\tau}$ strictly.

(ii) $v\left(a^{n}, \tau\right)>0$ and $v\left(b^{n}, \tau\right)>0$ strictly.

(iii) $v\left(a^{n}, \tau\right) \neq v\left(b^{s}, \tau\right)$ for $n, s \geqslant 1$,

(iv) $v\left(a^{n}, \tau\right), v\left(b^{n}, \tau\right) \notin C$ for $n \geqslant 1$.

Sequences satisfying (i) and (ii) exist by the definition of $a_{\tau}$ and $b_{\tau}$ and the continuity of $v$. There must be some point $x_{0} \in\left(a^{2}, a^{1}\right]$ where $v\left(x_{0}, \tau\right)>0$ and $v_{x}\left(x_{0}, \tau\right) \neq 0$, for otherwise $v_{x}(x, \tau) \equiv 0$ near $x=a^{1}$, and by induction $v_{x}(x, \tau) \equiv 0$ on $\left[a^{2}, a^{1}\right]$, which contradicts (i). Near $x=x_{0}$ the function $v(x, \tau)$ takes on a continuum of values, since $v_{x}\left(x_{0}, \tau\right) \neq 0$. Let $x_{0}^{\prime}$ be a point near $x_{0}$ such that $x_{0}^{\prime} \in\left(a^{2}, a^{1}\right), v\left(x_{0}^{\prime}, \tau\right)>0$ and $v\left(x_{0}^{\prime}, \tau\right) \notin C$. Then $x_{0}^{\prime}$ can serve as our new $a^{i}$ and by induction we arrive at a sequence $\left\{a^{n}\right\}$ such that (iv) is satisfied and $a^{n} \rightarrow a_{\tau}$. Hence (i), (ii) and (iv) are satisfied by some subsequence which we again denote $\left\{a^{n}\right\}$. A sequence $\left\{b^{n}\right\}$ satisfying (i), (ii) and (iv) is chosen similarly, and then subsequences can be chosen so that (iii) also holds.

By Lemma 5.4 and Remark 3.6 it follows that for arbitrary $n>1$ there exists a continuous curve $C_{n}^{a}$ lying in $P[u]$ connecting $\left(a^{n}, \tau\right)$ to $I$ along which the $t$-coordinate is decreasing. There is also a curve $C_{n}^{b}$ connecting $\left(b^{n}, \tau\right)$ to $I$. Let $D_{n}$ denote the domain bounded by $C_{n}^{a}, C_{n}^{b}$, and $I$. Because of (iii) these curves cannot intersect. Now consider the positive functions $w_{m}$ which are solutions of (3.1) and which converge to $\phi(u)$ in $\bar{G}$. For sufficiently large $m$, since $w_{m}>\phi(u)$, it follows that the functions $w_{m}$ must be bounded below by a positive constant uniformly with respect to $m$ on the lower and lateral boundary of $D_{n}$. But then it follows from the maximum principle that the functions $w_{m}$ are bounded below by a positive constant in $\bar{D}_{n}$, uniformly in $m$ $\left(m>m_{0}\right)$. Therefore, since the $w_{m}$ monotonically decrease to $\dot{\phi}(u)$ it follows that $v=\psi(u)$ must be positive in $\bar{D}_{n}$. This proves that $v>0$ on the line segment $a^{n}<x<b^{n}, 0<t<T$. Thus the assertion of the theorem is true with $\zeta_{1}(t)=a_{t}$ and $\zeta_{2}(t)=b_{t}$. The monotonicity of the curves $\zeta_{i}$ follows immediately from Theorem 4.3.

To prove the Lipschitz continuity $\zeta_{2}(t)$ for $t>\tau>0$ we consider $u(x, t)$ as a weak solution of the Cauchy problem on $R^{1} \times(\tau, T)$ with initial data $u(x, \tau)$. By Theorem 4.4

$$
|v(x, \tau)-v(y, \tau)| \leqslant \xi_{\tau}|x-y| \text { for } x, y \in R^{!} .
$$

Let $\eta(x, t)$ be given by

$$
\eta(x, t)=\xi_{r}\left(\xi_{r}(t-\tau)-\left(x-\zeta_{2}(\tau)\right)\right)^{+} .
$$

As we have already seen (see (1.28)), $\tilde{u}(x, t) \equiv \psi^{-1}(\eta(x, t))$ is a weak solution of the problem 


$$
\begin{aligned}
u_{t} & =\partial^{2} \phi(u) / \partial x^{2} \quad \text { on } R^{1} \times(\tau, T), \\
u\left(a_{1}, t\right)=\psi^{-1} \eta\left(a_{1}, t\right) & \text { for } \tau \leqslant t \leqslant T, \\
u(x, \tau) & =\psi^{-1} \eta(x, \tau) \quad \text { for } x \geqslant a_{1} .
\end{aligned}
$$

Clearly $\tilde{u}(x, \tau) \geqslant u(x, \tau)$ for $x \geqslant a_{1}$ and, since $\tilde{u}\left(a_{1}, t\right)$ is monotone increasing in $t$ and $u\left(a_{1}, t\right) \leqslant u\left(a_{1}, 0\right)$ for $\tau \leqslant t \leqslant T$, we also have $\tilde{u}\left(a_{1}, t\right)>$ $u\left(a_{1}, t\right)$ for $\tau \leqslant t<T$. Hence, according to Theorem 4.2, $\tilde{u}(x, t) \geqslant u(x, t)$ and, therefore, $\eta(x, t) \geqslant v(x, t)$ on $\left[a_{1}, \infty\right) \times[\tau, T]$. Consequently, $\zeta_{2}(t)<$ $\zeta_{2}(\tau)+\xi_{r}(t-\tau)$ for $\tau \leqslant t \leqslant T$ and since, by the monotonicity of $\zeta_{2}(t)$, $0<\zeta_{2}(t)-\zeta_{2}(\tau)$ for $t \in[\tau, T]$, the Lipschitz continuity of $\zeta_{2}(t)$ in $[\tau, T]$ has been established. The proof for $\zeta_{1}$ is similar. Also, if $v_{0} \in C^{0,1}\left(R^{1}\right)$, the very same argument extends to give $\zeta_{i} \in C^{0,1}[0, T](i=1,2)$.

To prove that $\lim \zeta_{i}(t)=a_{i}(i=1,2)$ as $t \rightarrow 0$ note that for any $\varepsilon>0$ we can find functions $u_{0}^{\prime}$ and $u_{0}^{\prime \prime}$ such that $\psi\left(u_{0}^{\prime}\right)$ and $\psi\left(u_{0}^{\prime \prime}\right)$ are Lipschitz continuous, $u_{0}^{\prime}$ and $u_{0}^{\prime \prime}$ satisfy (2.8) with $I$ replaced by $I^{\prime}$ and $I^{\prime \prime}$, where $I^{\prime}=\left(a_{1}+\varepsilon, a_{2}-\varepsilon\right)$ and $I^{\prime \prime}=\left(a_{1}-\varepsilon, a_{2}+\varepsilon\right)$, and such that $u_{0}^{\prime}<u_{0}<u_{0}^{\prime \prime}$. Hence it follows from Theorem 4.1 that the corresponding weak solutions $u^{\prime}$ and $u^{\prime \prime}$ must satisfy the inequality $u^{\prime} \leqslant u \leqslant u^{\prime \prime}$ in $\bar{G}$. Since $v_{0}^{\prime}$ and $v_{0}^{\prime \prime}$ are Lipschitz continuous the interface curves for these functions are continuous at $t=0$. Hence $\left|\zeta_{i}(0)-a_{i}\right| \leqslant 2 \varepsilon$ and the result follows by the arbitrariness of $\varepsilon$. This concludes the proof.

Theorem 6.1, except for the Lipschitz continuity of the interface, was proved by Kalashnikov [9] in the case $\phi(u)=u^{m}$. The proof given here is quite different. The proof of the Lipschitz continuity is essentially the same as Aronson's [1] proof in the case $\phi(u)=u^{m}$.

VII. The interface equation. In this section, the functions $\psi$ and $\sigma$ are, as usual, defined by (1.14) and (1.18). Also $v(x, t) \equiv \psi(u(x, t))$ and $v_{0}(x) \equiv$ $\psi\left(u_{0}(x)\right)$.

LEMMA 7.1. Let $u(x, t)$ be a weak solution of (1.6), (1.7) with the initial data $u_{0}$ satisfying (1.8). Suppose $\phi \in \Phi_{0}$ and $\sigma^{\prime \prime}(s) \leqslant 0$ for $0<s \leqslant \sup v$. If $v_{0}^{\prime} \in$ $C\left[a_{1}, a_{2}\right]$ and if $v_{0}^{\prime}$ is absolutely continuous and ess $\inf _{I} v_{0}^{\prime \prime}>-\alpha$ for some constant $\alpha \geqslant 0$, then $v_{x x} \geqslant-\beta$ in $P[u]$ for some constant $\beta>0$.

Proof. As in the proof of Lemma 2 [1], it is possible to construct a sequence of positive bounded $C^{\infty}$ functions $v_{0 n}$ which satisfy:

(i) $v_{0 n}>v_{0}$ strictly in $R^{1}$,

(ii) $\left|v_{0 n}^{\prime}\right|<\sup _{I}\left|v_{0}^{\prime}\right|+1$ on $R^{1}$,

(iii) $v_{0 n}^{\prime \prime}(x) \geqslant-(\alpha+1)$ on $R^{1}$.

We then define the functions $\bar{v}_{0 n}$ which agree with the functions $v_{0 n}$ when $|x| \leqslant n-2$, which equal sup $v_{0 n}=M_{n}^{\prime}$ when $|x| \geqslant n-1$ and which otherwise satisfy $v_{0 n} \leqslant \bar{v}_{0 n} \leqslant M_{n}^{\prime}$. Set $\bar{w}_{0}^{n}=\left(\phi \circ \psi^{-1}\right)\left(\bar{v}_{0 n}\right)$ and note that these 
functions satisfy all of the requirements stated in the construction of $u$ (see the paragraph containing (3.1)). The corresponding functions $w_{n}$ which are solutions of (3.1) converge in $\bar{G}$ to $\phi(u)$. The functions $v_{n} \equiv(\psi \circ \Phi)\left(w_{n}\right)$ are solutions of the initial-boundary value problem

$$
\begin{aligned}
v_{t} & =\sigma(v) v_{x x}+v_{x}^{2}, \quad G_{n}, \\
v(x, 0) & =\bar{v}_{0 n}(x), \quad|x| \leqslant n, \\
v( \pm n, t) & =M_{n}^{\prime}, \quad t \in[0, T] .
\end{aligned}
$$

Clearly $v_{t}( \pm n, t)=0$ and therefore

$$
\frac{\partial^{2}}{\partial x^{2}} v_{n}( \pm n, t)=\frac{-\left((\partial / \partial x) v_{n}( \pm n, t)\right)^{2}}{\sigma\left(M_{n}^{\prime}\right)}
$$

For sufficiently large $n$ the numbers $M_{n}^{\prime}=\sup v_{0 n}$ satisfy $\sup v_{0} \leqslant M_{n}^{\prime} \leqslant$ sup $v_{0}+1$. In the proof of Theorem 3.1 it was shown that the functions $\left(\partial w_{n} / \partial x\right)( \pm n, t)$ are bounded uniformly in $n$. Since $v_{n}=(\psi \circ \Phi)\left(w_{n}\right)$ it follows from (1.16) that $\left(w_{n}\right)_{x}=u_{n}\left(v_{n}\right)_{x}$, and therefore the functions $\left(\partial v_{n} / \partial x\right)( \pm n, t)$ are bounded uniformly with respect to $n$. Hence

$$
\left|\partial^{2} v_{n} / \partial x^{2}\right| \leqslant c^{\prime} \quad \text { on } x= \pm n, \quad 0 \leqslant t \leqslant T
$$

where $c^{\prime}$ does not depend on $n$.

It is not difficult to see that the functions $\partial^{2} v_{n} / \partial x^{2}$ are uniformly bounded below on $-n \leqslant x<n, t=0$. Indeed, the procedure of "rounding up", by which the functions $\bar{v}_{0 n}$ are obtained from the functions $v_{0 n}$, may be accomplished by the same construction for all $n$. Therefore

$$
\partial^{2} v_{n} / \partial x^{2} \geqslant-\gamma \quad \text { on } t=0, \quad|x| \leqslant n,
$$

for some constant $\gamma>0$.

If we differentiate (7.1) twice with respect to $x$, and suppress the subscript $n$, we see that $P=\partial^{2} v_{n} / \partial x^{2}$ satisfies

$$
\begin{gathered}
\sigma(v) P_{x x}+2 v_{x}\left(\sigma^{\prime}(v)+1\right) P_{x}+\sigma^{\prime \prime}(v) v_{x}^{2} P-P_{t} \\
=-\left(\sigma^{\prime}(v)+2\right) P^{2} \leqslant 0 .
\end{gathered}
$$

It follows from (7.4) and (7.5) that, for some $\beta>0, P>-\beta$ on the lower and lateral boundary of $G_{n}$. For fixed $n, P$ can be viewed as a solution of the parabolic inequality (7.6) with continuous coefficients and a nonpositive $P$-coefficient. It therefore follows from the maximum principle that $P \geqslant-\beta$ in $G_{n}$, i.e.,

$$
\partial^{2} v_{n} / \partial x^{2} \geqslant-\beta, \quad G_{n}
$$

Since $\beta$ does not depend on $n$ and since $\partial^{2} v_{n} / \partial x^{2} \rightarrow \partial^{2} v / \partial x^{2}$ in $P[u]$ it follows that 


$$
v_{x x}>-\beta, \quad P[u] .
$$

Aronson [1] proved a similar result in the case $\phi(u)=u^{m}$.

THEOREM 7.2. Suppose the hypotheses of Lemma 7.1 hold. Then the quantities

$$
\begin{aligned}
v_{x}\left(\zeta_{i}(t), t\right) & \equiv \lim _{\substack{x \rightarrow \zeta_{i}(t) \\
(x, t) \in P[u]}} v_{x}(x, t) \quad(i=1,2), \\
\zeta_{i}^{\prime}(t) & \equiv \lim _{\Delta t \rightarrow 0^{+}} \frac{\zeta_{i}(t+\Delta t)-\zeta_{i}(t)}{\Delta t} \quad(i=1,2)
\end{aligned}
$$

exist for all $t \geqslant 0$, and, for all $t \geqslant 0$,

$$
\zeta_{i}^{\prime}(t)=-v_{x}\left(\zeta_{i}(t), t\right) \quad(i=1,2) .
$$

Proof. Since $v_{x x} \geqslant-\beta$ in $P[u]$, the function $f(x, t)=v(x, t)+\beta x^{2}$ satisfies $f_{x x}>0$ in $P[u]$. Hence $f_{x}(x, t)$ is a monotone increasing function of $x$ for each fixed $t$. Since $v_{x}$ is bounded in $P[u]$ (Theorem 5.4) it follows that

$$
\lim _{\substack{x \rightarrow \zeta_{1}(t) \\(x, t) \in P[u]}} f_{x}(x, t) \quad(i=1,2)
$$

exists and is finite for all $t$. Consequently,

$$
\lim _{\substack{x \rightarrow \zeta_{i}(t) \\(x, t) \in P[u]}} v_{x}(x, t) \quad(i=1,2)
$$

exists and is finite for each fixed $t$.

Set $a=\zeta_{2}(0)$ and $v_{x}(a, 0)=\alpha$. Then either $\alpha=0$ or $\alpha<0$ and we shall consider these two cases separately.

CASE 1. $\alpha<0$. We shall show that for any sufficiently small $\varepsilon>0$ there exists a $\delta>0$ depending on $\varepsilon$ only such that $\left|\left(\zeta_{2}(\Delta t)-a\right) / \Delta t+\alpha\right|<\varepsilon$, whenever $0<\Delta t<\delta$. Since $\lim _{x \rightarrow a-} v_{x}(x, 0)=\alpha$, it follows that for any $0<\varepsilon<-\alpha$ there exists a $\delta_{0}>0$ such that $\alpha-\varepsilon<v_{x}(x, 0)<\alpha+\varepsilon$ whenever $a-\delta_{0}<x<a$. Using the mean value theorem we get

$$
(\alpha+\varepsilon)(x-a)<v(x, 0)<(\alpha-\varepsilon)(x-a)
$$

whenever $a-\delta_{0}<x<a$. We define functions $v_{1}, v_{2}, u_{1}, u_{2}$ by:

$$
\begin{aligned}
& v_{1}(x, t)=\left\{\begin{array}{l}
(\alpha+\varepsilon)[(\alpha+\varepsilon) t+(x-a)] \\
0 \text { if } x>a-(\alpha+\varepsilon) t ;
\end{array} \text { if } x<a-(\alpha+\varepsilon) t,\right. \\
& v_{2}(x, t)=\left\{\begin{array}{l}
(\alpha-\varepsilon)[(\alpha-\varepsilon) t-(x-a)] \text { if } x<a-(\alpha-\varepsilon) t, \\
0 \text { if } x>a-(\alpha-\varepsilon) t ;
\end{array}\right. \\
& u_{1}(x, t)=\psi^{-1}\left(v_{1}(x, t)\right), \\
& u_{2}(x, t)=\psi^{-1}\left(v_{2}(x, t)\right) .
\end{aligned}
$$


We have already seen (see (1.28)) that $u_{1}$ and $u_{2}$ are weak solutions, as defined in [14], of the problem

$$
\begin{aligned}
\left(u_{i}\right)_{t} & =\left(\phi\left(u_{i}\right)\right)_{x x} \quad\left(\left(a-\delta_{0}, \infty\right) \times(0, T)\right), \\
u_{i}(x, 0) & =\psi^{-1}\left(v_{i}(x, 0)\right) \quad\left(a-\delta_{0} \leqslant x<\infty\right), \\
u_{i}\left(a-\delta_{0}, t\right) & =\psi^{-1}\left(v_{i}\left(a-\delta_{0}, t\right)\right) \quad(0<t \leqslant T) .
\end{aligned}
$$

From (7.9) it follows that

$$
v_{1}(x, 0)<v(x, 0)<v_{2}(x, 0) \text { for } a-\delta_{0}<x
$$

and, consequently,

$$
u_{1}(x, 0) \leqslant u(x, 0) \leqslant u_{2}(x, 0) \text { for } a-\delta_{0} \leqslant x .
$$

By continuity there exists $\tau_{1}>0$ such that

$$
v_{1}\left(a-\delta_{0}, t\right)<v\left(a-\delta_{0}, t\right) \quad\left(0<t<\tau_{1}\right) .
$$

Therefore Theorem 4.2 yields

$$
v(x, t) \geqslant v_{1}(x, t) \text { in }\left[a-\delta_{0}, \infty\right) \times\left[0, \tau_{1}\right]
$$

and from this it follows that

$$
\zeta_{2}(t)>a-(\alpha+\varepsilon) t \quad\left(0<t<\tau_{1}\right)
$$

or

$$
\left(\zeta_{2}(t)-a\right) / t+\alpha>-\varepsilon \quad\left(0<t<\tau_{1}\right) .
$$

Similarly it follows that there exists $\tau_{2}>0$ such that

$$
\left(\zeta_{i}(t)-a\right) / t+\alpha<\varepsilon \quad\left(0<t<\tau_{2}\right) .
$$

If we set $\delta=\min \left(\tau_{1}, \tau_{2}\right)>0$ we get

$$
\left|\left(\zeta_{2}(t)-a\right) / t+\alpha\right| \leqslant \varepsilon \quad(0<t<\delta)
$$

which completes the proof of Case 1 .

CASE 2. $\alpha=0$. We can define $u_{2}(x, t)$ as above to obtain (7.14) which, since $\alpha=0$, assumes the form

$$
\left(\zeta_{2}(t)-a\right) / t<\varepsilon \quad\left(0<t<\tau_{2}\right) .
$$

By the monotonicity of $\zeta_{2}$ we have

$$
\left(\zeta_{2}(t)-a\right) / t \geq 0 \quad(t>0)
$$

We therefore merely choose $\delta=\tau_{2}>0$ to complete the proof of Case 2 .

REMARK 7.3. We have already shown in Theorem 6.1 that the $\zeta_{i}(t)$ are Lipschitz in $[0, T]$ and thus by Theorem $7.2 \zeta_{i}^{\prime}(t)=-v_{x}\left(\zeta_{i}(t), t\right)(i=1,2)$ holds a.e. if we interpret the derivatives $\zeta_{i}^{\prime}(t)$ in the usual sense.

It is interesting to note that this weaker result follows by resorting to the contour integral equation 


$$
\int_{L} u d x+\frac{\partial}{\partial x} \phi(u) d t=0
$$

of Kalashnikov [9], which holds for any piecewise smooth loop $L$ in $G$, and is proved by integrating (1.6) by parts. We assume for simplicity that $v_{0}(x)$ is monotone near $a_{1}$ and $a_{2}$ and that the other conditions of Theorem 7.2 are satisfied. Let $0<\tau<T$. For sufficiently small $\varepsilon_{0}>0$, Lemma 5.4 and Remark 5.6 guarantee the existence of a level curve $x=\xi_{\varepsilon}(t) \in C[0, \tau] \cap$ $C^{\prime}(0, \tau)$ for some $0<\varepsilon<\varepsilon_{0}$ lying in $P[u]$ along which $u \equiv \varepsilon$ and such that $0<u<\varepsilon$ on the line segments $\xi_{\varepsilon}(0)<x<a_{2}, t=0$, and $\xi_{e}(\tau)<x<$ $\zeta_{2}(\tau), t=\tau$. It follows from (1.16) that $\partial \phi(u) / \partial x=u v_{x}$ in $P[u]$ and, consequently, (7.18) applied to the loop $L_{e}$ bounded above by $t=\tau$, below by $t=0$ and laterally by $\xi_{e}$ and $\zeta_{2}$ yields

$$
\int_{L_{e}} u\left(d x+v_{x} d t\right)=0
$$

or

$$
\int_{\xi_{e}(\tau)}^{\zeta_{2}(\tau)} u(x, \tau) d x-\int_{\xi_{e}(0)}^{a_{2}} u_{0}(x) d x+\varepsilon \int_{\xi_{\varepsilon}}\left(d x+v_{x} d t\right)=0 .
$$

Thus

$$
\begin{aligned}
\xi_{\varepsilon}(\tau)-\xi_{\varepsilon}(0) & +\int_{0}^{\tau} v_{x}\left(\xi_{\varepsilon}(t), t\right) d t \\
= & \int_{\xi_{e}(0)}^{a_{2}} \frac{u_{0}(x)}{\varepsilon} d x-\int_{\xi_{e}(\tau)}^{\xi_{2}(\tau)} \frac{u(x, \tau)}{\varepsilon} d x .
\end{aligned}
$$

Since, as in the proof of Theorem 7.2, $\lim _{x \rightarrow \zeta_{2}(t)} v_{x}(x, t) \equiv v_{x}\left(\zeta_{2}(t), t\right)$ exists, and since $\varepsilon$ may be chosen arbitrarily small, we can use the Lebesgue bounded convergence theorem and let $\varepsilon \rightarrow 0$ in (7.19) to conclude

$$
\zeta_{2}(\tau)=a_{2}-\int_{0}^{\tau} v_{x}\left(\zeta_{2}(t), t\right) d t
$$

which implies

$$
\zeta_{2}^{\prime}(t)=-v_{x}\left(\zeta_{2}(t), t\right) \text { for a.e. } t \in(0, T) .
$$

VIII. The initial growth of the interface curves. In this section $u(x, t)$ is a weak solution of (1.6), (1.7) and $u_{0}(x)$ satisfies (1.8). As usual, $\phi \in \Phi_{0}, v=$ $\psi(u)$ and $v_{0}=\psi\left(u_{0}\right)$ and $\sigma$ is defined in (1.18).

THEOREM 8.1. If $v_{0}(x)>\left(a_{2}-x\right)^{\gamma}$ for $a_{2}-\delta<x<a_{2}$ where $0<\delta<1$ and $\gamma<2$ are constants then $v\left(a_{2}, t\right)>0$ for all $t>0$. The analogous result holds at $x=a_{1}$.

Proof. Without loss of generality, $1<\gamma<2$ and $v_{0}(x) \equiv\left(a_{2}-x\right)^{\gamma}$ near $a_{2}$. Let $a=a_{2}-\delta / 2$ and choose $\tilde{x} \in\left(a, a_{2}\right)$ so that 


$$
v_{0}^{\prime}(\tilde{x})=-\gamma\left(a_{2}-\tilde{x}\right)^{\gamma-1}=\frac{1}{2} v_{0}(a) /\left(a-a_{2}\right) .
$$

Set $x_{1}=\tilde{x}$ and define $\left\{x_{n}\right\}$ recursively by $x_{n+1}=\frac{1}{2}\left(x_{n}+a_{2}\right)$ for $n \geq 1$. The tangent line $T_{n}$ at $\left(x_{n}, v_{0}\left(x_{n}\right)\right)$ is given by $T_{n}: y=v_{0}\left(x_{n}\right)+\alpha_{n}\left(x-x_{n}\right)$ where $\alpha_{n} \equiv v_{0}^{\prime}\left(x_{n}\right)=-\gamma\left(a_{2}-x_{n}\right)^{\gamma-1}$. Then the $x$-intercept $\tilde{x}_{n}$ of $T_{n}$ is given by $\tilde{x}_{n}=x_{n}+v_{0}\left(x_{n}\right) /\left(-\alpha_{n}\right)$ and the intersection $\left(a, b_{n}\right)$ of the line $x=a$ and $T_{n}$ is given by $b_{n}=v_{0}\left(x_{n}\right)+\alpha_{n}\left(a-x_{n}\right)$. Since $v_{0}^{\prime \prime}(x)=\gamma(\gamma-1)\left(a_{2}-x\right)^{\gamma-2}>$ 0 for $x \in\left[a, a_{2}\right)$, it follows that $v_{0}$ never falls below the line $T_{n}$ on $\left[a, a_{2}\right)$. From this, one easily deduces that $b_{n} \backslash$. Since

$$
v_{t}(a, 0)=\sigma(v)\left[\gamma(\gamma-1)\left(a_{2}-a\right)^{\gamma-2}\right]+\gamma^{2}\left(a_{2}-a\right)^{2 \gamma-2}
$$

it follows that $v_{t}(a, 0)>0$ and by continuity $v_{t}(a, t)>0$ for $t \in[0, \tau]$, for some $\tau>0$.

Now consider a family of functions $\left\{f_{n}\right\}$ given by

$$
f_{n}(x, t)= \begin{cases}\alpha_{n}^{2} t+\alpha_{n}\left(x-\tilde{x}_{n}\right) & \text { if } x<\tilde{x}_{n}-\alpha_{n} t, \\ 0 & \text { if } x>\tilde{x}_{n}-\alpha_{n} t .\end{cases}
$$

Note that $f_{n}(x, 0)$ coincides with $T_{n}$ when $x<\tilde{x}_{n}$ and that each function $\psi^{-1} \circ f_{n}$ may be regarded as a weak solution of the boundary value problem on $[a, \infty) \times[0, \infty)$ with the obvious boundary values. It is clear that $f_{n}\left(a_{2}, t\right)$ $=0$ for $0 \leqslant t \leqslant \tau_{n}$ and $f_{n}\left(a_{2}, t\right)>0$ for $t>\tau_{n}$ where $\tau_{n}=\left(a_{2}-\tilde{x}_{n}\right) /\left(-\alpha_{n}\right)$ $>0$. It is easy to compute

$$
\tau_{n}=\left((\gamma-1) / \gamma^{2}\right)\left(a_{2}-x_{n}\right)^{2-\gamma} \text {. }
$$

Since we assume $\gamma<2$ (and this is crucial) it follows that $\lim \tau_{n}=0$ as $n \rightarrow \infty$ and (by deleting a finite number of $n$ ) we may assume that $\tau_{n}<\tau$ for $n>1$. By direct computation

$$
f_{n}\left(a, \tau_{n}\right)=-\alpha_{n}\left[a_{2}-a\right]
$$

and since $\alpha_{1}<\alpha_{n}$ it follows that

$$
f_{n}\left(a, \tau_{n}\right)<-\alpha_{1}\left[a_{2}-a\right]=v_{0}^{\prime}(\tilde{x})\left(a-a_{2}\right)
$$

and, consequently, by our choice of $\tilde{x}, f_{n}\left(a, \tau_{n}\right)<\frac{1}{2} v_{0}(a)$. Therefore, since each $f_{n}$ is monotone in $t$, there exist constants $\varepsilon_{n}>0$ such that

$$
f_{n}(a, t)<v_{0}(a)<v(a, t), \quad 0 \leqslant t \leqslant \tau_{n}+\varepsilon_{n}<\tau .
$$

It follows by Theorem 4.2 that

$$
f_{n}(x, t) \leqslant v(x, t) \text { in }[a, \infty) \times\left[0, \tau_{n}+\varepsilon_{n}\right] .
$$

But $f_{n}\left(a_{2}, t\right)>0$ for $\tau_{n}<t \leqslant \tau_{n}+\varepsilon_{n}$ and, consequently, $v\left(a_{2}, t\right)>0$ for $\tau_{n}<t<\tau_{n}+\varepsilon_{n}$. By Theorem 4.3, $v\left(a_{2}, t\right)>0$ for $t>\tau_{n}$, and since $\tau_{n}>0$, this establishes the result.

THEOREM 8.2. If $v_{0}(x) \leqslant b\left(x-a_{2}\right)^{2}$ for $a_{2}-\delta<x<a_{2}$ for some constants 
$b, \delta>0$ then there exists a constant $\tau>0$ such that $\zeta_{2}(t)=a_{2}$ for $0<t<$ $\tau$.

The analogous result holds for $\zeta_{1}(t)$.

Proof. Let $B=\sup \left\{\sigma^{\prime}(s) \mid 0<s<1+\sup v\right\}$. Then

$$
0<\sigma(s)<B s \text { for } 0<s<1+\sup v \text {. }
$$

It suffices to consider the case $a_{2}=0$, and then

$$
v_{0}(x)=b x^{2} \text { for }-\delta<x<0 .
$$

Let $0<t_{3}<t_{2}$ and define

$$
\begin{aligned}
& R=(-\delta / 2, \delta / 2) \times\left(0, t_{3}\right), \\
& B=[-\delta / 2, \delta / 2], \\
& S=\left\{(x, t) \mid x= \pm \delta / 2,0 \leqslant t<t_{3}\right\} .
\end{aligned}
$$

Let $\left\{w_{n}\right\}$ be the solutions of (3.1) encountered in the construction of $u$. Letting $M=\sup u$, we know there exist constants $\alpha_{n}>0$ such that

$$
\begin{gathered}
0<\alpha_{n}<w_{n} \leqslant \phi(M)+1, \quad \text { in } \bar{R}, \\
w_{n}>w \text { in } \bar{R} .
\end{gathered}
$$

The functions $v_{n}=(\psi \circ \Phi)\left(w_{n}\right)$ satisfy

$$
\begin{gathered}
\frac{\partial}{\partial t} v_{n}=\sigma\left(v_{n}\right) \frac{\partial^{2}}{\partial x^{2}} v_{n}+\left(\frac{\partial}{\partial x} v_{n}\right)^{2}, \quad(x, t) \in R, \\
v_{n}>v, \quad \bar{R}, \\
0<\beta_{n}<v_{n}<\bar{M} \equiv(\psi \circ \Phi)(\phi(M)+1), \quad \bar{R},
\end{gathered}
$$

for some constants $\beta_{n}$.

It is not difficult to verify that the function

$$
\bar{v}(x, t)=C x^{2} /\left(t_{2}-t\right) \text { for }(x, t) \in R^{1} \times\left(0, t_{3}\right),
$$

where $C=1 / 2(b+2)$ satisfies the equation

$$
\bar{v}_{t}=\bar{\sigma}(\bar{v}) \bar{v}_{x x}+\bar{v}_{x}^{2} \text { for }(x, t) \in R,
$$

where $\bar{\sigma}(s)=B s$.

Now we shall choose $t_{2}$ and $t_{3}$ smaller if necessary so that

$$
\bar{v}( \pm \delta / 2,0)>\bar{M}+1 \text {. }
$$

We define functions $\bar{v}_{n}$ by

$$
\bar{v}_{n}(x, t)=\bar{v}(x, t)+c_{n},
$$

where $c_{n}>0$ are chosen such that

$$
B c_{n}<\min _{\beta_{n}<\theta<\bar{M}}[B \theta-\sigma(\theta)]
$$


and $c_{n}>0$ strictly. For future reference we state

$$
\sigma\left(v_{n}\right) \leqslant \bar{\sigma}\left(v_{n}\right)-\bar{\sigma}\left(c_{n}\right) \text { in } \bar{R} .
$$

The functions $\bar{v}_{n}$ satisfy the equation

$$
\left(\bar{v}_{n}\right)_{t}=\bar{\sigma}\left(\bar{v}_{n}\right)\left(\bar{v}_{n}\right)_{x x}+\left(\frac{\partial}{\partial x} \bar{v}_{n}\right)^{2}-\bar{\sigma}\left(c_{n}\right) \bar{v}_{x x}^{n} \text { in } R .
$$

If we subtract (8.4) from (8.11) and define $P^{n}=\bar{v}_{n}-v_{n}$ we get

$$
\begin{gathered}
\sigma\left(v_{n}\right) P_{x x}^{n}+\left(\left(\bar{v}_{n}\right)_{x}+\left(v_{n}\right)_{x}\right) P_{x}^{n}+B\left(\bar{v}_{n}\right)_{x x} P^{n}-P_{t}^{n} \\
=\left(\bar{v}_{n}\right)_{x x}\left[\bar{\sigma}\left(c_{n}\right)-\bar{\sigma}\left(v_{n}\right)+\sigma\left(v_{n}\right)\right]
\end{gathered}
$$

in $R$. It can be computed directly that $\left(\bar{v}_{n}\right)_{x x} \geqslant 0$ in $R$, and this fact, together with (8.10) and (8.12), gives, in $R$,

(8.13) $L_{n}\left[P^{n}\right] \equiv \sigma\left(v_{n}\right) P_{x x}^{n}+\left(\left(\bar{v}_{n}\right)_{x}+\left(v_{n}\right)_{x}\right) P_{x}^{n}+B\left(\bar{v}_{n}\right)_{x x} P-P_{t}<0$.

From (8.6), (8.8) and the easily verified fact that $\left(\bar{v}_{n}\right)_{t}>0$ in $\bar{R}$, we see that

$$
\bar{v}_{n} \geqslant v_{n} \text { on } S \text {. }
$$

Since by (8.5) $v_{n} \succ v$ in $\bar{R}$, and since by (8.9) $\bar{v}_{n}>\bar{v}$ strictly in $\bar{R}$, we can extract a subsequence of $\left\{v_{n}\right\}$, which we again denote by $\left\{v_{n}\right\}$, such that

$$
\bar{v}_{n} \geqslant v_{n} \text { on } B \text {. }
$$

Using (8.6) we see that for fixed $n$ the $L_{n}$ in (8.13) are uniformly parabolic operators with bounded coefficients. The maximum principle applied to (8.13)-(8.15) in $R$ implies $\bar{v}_{n}>v_{n}$ in $\bar{R}$. Letting $n \rightarrow \infty$ we get

$$
\bar{v} \geqslant v \text { in } \bar{R} \text {. }
$$

We conclude the proof of the theorem by taking $\tau=t_{3}$.

Kalashnikov proved Theorem 8.2 in the case $\phi(u)=u^{m}, m>1$, also by comparison with the same function $\bar{v}(x, t)$.

THEOREM 8.3. Suppose that for some constants $x_{0}, A>0$ we have $v_{0}(x)>A$ when $|x| \leqslant x_{0}$. Let $B=\sup \left\{\sigma^{\prime}(s) \mid 0<s<1+\sup v\right\}$, so that (8.1) holds. Then there exist positive constants $\theta_{1}, \theta_{2}$ and $\theta_{3}$ depending only on $B$ such that

$$
\zeta_{2}(t)-x_{0} \geqslant \sqrt{A} \theta_{1} \sqrt{t} \text { when } 0<t<\tau_{0}
$$

and

$$
-x_{0}-\zeta_{1}(t)>\sqrt{A} \theta_{2} \sqrt{t} \text { when } 0 \leqslant t \leqslant \tau_{0}
$$

where $\tau_{0}=\left(x_{0}^{2} / A\right) \theta_{3}>0$.

Proof. We shall consider only the case $i=2$ since the proof is similar if $i=1$. Equation (1.24) gives a weak solution $u(x, t)$ of $(1.22),(1.7)$ with $u_{0}$ given by (1.25). According to (1.20) when $m=B+1$, where $B$ occurs in 
(8.1), the transformed function $\beta \equiv \psi(u)$ is given by

$$
\beta(x, t)=\frac{B+1}{B}\left\{\begin{array}{l}
\lambda^{-B}(t)\left(1-x^{2} / \lambda^{2}(t)\right) \text { if }|x|>\lambda(t), \\
0 \text { if }|x| \geqslant \lambda(t) .
\end{array}\right.
$$

For $K, L>0$ we define

$$
\beta_{K, L}(x, t)=K \beta\left(L x, K L^{2} t\right)
$$

and note that $u_{K, L} \equiv \psi^{-1} \beta_{K, L}$ is a weak solution of (1.22) with the obvious initial data. If we set $K=A C^{B}(B / B+1) \equiv \bar{C}$, we generate a family $\left\{\beta_{L}\right\}$ of functions,

$$
\beta_{L}(x, t)=\bar{C} \beta\left(L x, \bar{C} L^{2} t\right)
$$

Observe:

$$
\begin{gathered}
\beta_{L}(0,0)=A, \\
\beta_{L}(x, t)=0 \text { iff }|x| \geqslant L^{-1} \lambda\left(\bar{C} L^{2} t\right) \equiv \lambda^{L}(t) \\
\left(\beta_{L}(x, 0)=0 \text { iff }|x| \geqslant C / L\right), \\
\partial^{2} / \partial x^{2} \beta_{L}(x, t) \leqslant 0 \text { if }|x|<\lambda^{L}(T),
\end{gathered}
$$

(8.22) for fixed $L,\left|(\partial / \partial x) \beta_{L}\right|$ is bounded for $|x|<\lambda^{L}(t)$ and $0 \leqslant t \leqslant T$.

We will henceforth restrict our attention to $L$ satisfying

$$
L \geqslant C / x_{0} \text {. }
$$

Define in $G$ the functions $\hat{\beta}_{L}$ given by

$$
\hat{\beta}_{L}(x, t)=\beta_{L}\left(x-x_{0}+C / L, t\right)
$$

and notice

(8.24)

$$
\hat{\beta}_{L}(x, t)=0 \text { if } x-x_{0} \geqslant \lambda^{L}(t)-C / L .
$$

Denote by $\Sigma$ the set in $t>0$, where $\hat{\beta}_{L}>0$, i.e.

$$
\Sigma=\left\{(x, t)|| x-\left(x_{0}-C / L\right) \mid<\lambda^{L}(t), t>0\right\} .
$$

Let $S^{*}$ denote the lateral boundary of $\Sigma$ and let $B^{*}$ denote the lower boundary, i.e.

$$
B^{*}=\left\{(x, 0) \mid x_{0}-2 C / L<x<x_{0}\right\}
$$

Recall that $v$ is the limit of a monotone decreasing sequence $\left\{v_{n}\right\}$ of positive solutions of

$$
\left(v_{n}\right)_{t}=\sigma\left(v_{n}\right)\left(v_{n}\right)_{x x}+\left(v_{n}\right)_{x}^{2} \text { for }(x, t) \in g_{n} .
$$

It is easily verified that $\hat{\beta}_{L}$ satisfies

$$
\hat{\beta}_{t}=B \hat{\beta} \hat{\beta}_{x x}+\hat{\beta}_{x}^{2} \text { for }(x, t) \in \Sigma
$$


where we have suppressed, and will continue to suppress, the subscript $L$ as long as it remains constant. Because $v_{n}>0$ in $G_{n}$ it follows that $v_{n}>\hat{\beta}$ on $S^{*}$, and since $v_{n}>$ it follows that $v_{n}>\hat{\beta}$ on $B^{*}$. Hence

$$
v_{n}>\hat{\beta} \text { on } B^{*} \cup S^{*} \text {. }
$$

Subtracting (8.25) from (8.26) we get, after setting $P^{n}=\hat{\beta}-v_{n}$,

$$
\begin{aligned}
L_{n}\left(P^{n}\right) & =\sigma\left(v_{n}\right) P_{x x}^{n}+\left(\hat{\beta}_{x}+\left(v_{n}\right)_{x}\right) P_{x}^{n}+B \hat{\beta}_{x x} P^{n}-P_{t}^{n} \\
& =\left[\sigma\left(v_{n}\right)-B v_{n}\right] \hat{\beta}_{x x} \text { in } \Sigma .
\end{aligned}
$$

Since $\hat{\beta}_{x x}<0$ in $\Sigma$ and since, by hypothesis,

$$
\sigma(\theta) \leqslant B \theta \text { for } 0<\theta<\infty,
$$

we get

$$
L_{n}\left(P^{n}\right) \geqslant 0 \text { in } \Sigma \text {. }
$$

For fixed $n,\left|\left(v_{n}\right)_{x}\right|$ is bounded in $G_{n}$. Also $\left|\hat{\beta}_{x}\right|$ is bounded in $\Sigma$ and, as mentioned before, $\hat{\beta}_{x x} \leqslant 0$ in $\Sigma$. The maximum principle may be applied to (8.27), (8.29) to yield

$$
P^{n}=\hat{\beta}-v_{n} \leqslant 0 \text { in } \Sigma .
$$

Passing to the limit as $n \rightarrow \infty$ and recalling that $\beta=\beta_{L}$, we get

$$
\hat{\beta}_{L}<v \text { in } \Sigma \text {. }
$$

Consequently, $v$ is positive whenever $\hat{\beta}_{L}$ is positive and we get

$$
\zeta_{2}(t) \geqslant \lambda^{L}(t)+x_{0}-C / L \text { for } t \geqslant 0
$$

or

$$
\zeta_{2}(t)-x_{0} \geqslant f(L, t) \text { for } t \geqslant 0, L \geqslant C / x_{0},
$$

where $f(L, t) \equiv \lambda^{L}(t)-C / L$.

It is not difficult to see that the function

$$
g(w)=-B w^{B+2}+(B+2) w^{B+1}-2
$$

has precisely two zeros for $0<w<\infty$, one zero at $w=1$ and the other at $w=w_{0}$ where

$$
(B+1) / B<w_{0}<(B+2) / B .
$$

Indeed $g$ increases for $0<w<(B+1) / B$ and decreases to $-\infty$ as $w \rightarrow \infty$ for $w>(B+1) / B$.

The second inequality in (8.33) follows from

$$
-B w_{0}^{B+2}+(B+2) w_{0}^{B+1}=2>0 .
$$

If we define

$$
L_{0} \equiv \sqrt{\left(w_{0}^{B+2}-1\right) / \bar{C} t} \text { and } \tau_{0} \equiv\left(w_{0}^{B+2}-1\right)\left(x_{0}^{2} / \bar{C} C^{2}\right),
$$


then it follows that

$$
L_{0} \geqslant C / x_{0} \text { for } 0<t \leqslant \tau_{0}
$$

and, by (8.32),

$$
\zeta_{2}(t)-x_{0} \geqslant f\left(L_{0}, t\right) \text { for } 0<t<\tau_{0}
$$

By direct computation we get

$$
f\left(L_{0}, t\right)=\left(C / L_{0}\right)\left(w_{0}-1\right)=C\left(w_{0}-1\right) \sqrt{\bar{C} t /\left(w_{0}^{B+2}-1\right)}
$$

and this, together with (8.34), yields

(8.35) $\zeta_{2}(t) \geqslant x_{0}+\left(C\left(w_{0}-1\right) \bar{C}^{1 / 2} /\left(w_{0}^{B+2}-1\right)^{1 / 2}\right) \sqrt{t}$ for $0 \leqslant t \leqslant \tau_{0}$ which concludes the proof.

The motivation for the choice of $L_{0}$ in the proof is, of course, that the value $L=L_{0}$ maximizes $f(L, t)$ when $t$ is fixed and $0<t<\tau_{0}$. In fact, one can easily check that

$$
(B+2) w^{B+1} L^{2} / C \frac{\partial}{\partial L} f(L, t)=g(w)
$$

where $w=\left(\bar{C} L^{2} t+1\right)^{1 /(B+2)}$.

THEOREM 8.4. If $u_{0}$ satisfies (1.8) and if $N=\sup \psi\left(u_{0}\right)$ then

$$
\zeta_{2}(t) \leqslant a_{2}+2 \sqrt{N t} \text { for } t \geqslant 0
$$

and

$$
\zeta_{1}(t) \geqslant a_{1}-2 \sqrt{N t} \text { for } t \geqslant 0 \text {. }
$$

Proof. Consider for any real number $b>a_{2}$ the function

$$
v_{b}(x, t)= \begin{cases}\alpha_{b}^{2} t-\alpha_{b}(x-b) & \text { for } x<b+\alpha_{b} t \\ 0 & \text { for } x \geqslant b+\alpha_{b} t\end{cases}
$$

where $\alpha_{b}=N /\left(b-a_{2}\right)$. Then (see (1.28)) the function $u_{b} \equiv \psi^{-1} \circ v_{b}$ is a weak solution of the problem:

$$
\begin{aligned}
u_{t} & =[\phi(u)]_{x x} \quad \text { in }\left(a_{2}, \infty\right) \times(0, T), \\
u\left(a_{2}, t\right) & =\psi^{-1}\left(v_{b}\left(a_{2}, t\right)\right) \quad \text { for } t \geqslant 0, \\
u(x, 0) & =\psi^{-1}\left(v_{b}(x, 0)\right) \quad \text { for } x \geqslant a_{2} .
\end{aligned}
$$

Clearly,

$$
v_{0}(x)=\psi\left(u_{0}(x)\right)<N \chi\left[a_{1}, a_{2}\right](x) \leqslant v_{b}(x, 0) \text { for }-\infty<x<\infty
$$

where $\chi_{\left[a_{1}, a_{2}\right]}$ is the characteristic function on $\left[a_{1}, a_{2}\right]$. Hence, by the monotonicity of $\psi^{-1}$,

$$
u_{0}(x)=\psi^{-1}\left(v_{0}(x)\right) \leqslant \psi^{-1}\left(v_{b}(x, 0)\right) \text { for } x \in R^{1}
$$


Also, since $v(x, t)=\psi(u(x, t)) \leqslant N$ in $G$ and since, for fixed $x$, the mapping $t \rightarrow v_{b}(x, t)$ is monotone increasing, we get

$$
v\left(a_{2}, t\right)<N=v_{b}\left(a_{2}, 0\right) \leqslant v_{b}\left(a_{2}, t\right)
$$

and

$$
u\left(a_{2}, t\right) \leqslant \psi^{-1}\left(v_{b}\left(a_{2}, t\right)\right) \text { for } t \geqslant 0 .
$$

Theorem 4.2 enables us to conclude

$$
u(x, t) \leqslant u_{b}(x, t) \text { in }\left[a_{2}, \infty\right) \times[0, T]
$$

or

$$
v(x, t) \leqslant v_{b}(x, t) \text { in }\left[a_{2}, \infty\right) \times[0, T] .
$$

This last equation implies that

$$
\zeta_{2}(t) \leqslant \zeta_{b}(t) \equiv b+\alpha_{b} t \text { for } t \geqslant 0 .
$$

Choosing $b=a_{2}+\sqrt{N t}$ (which minimizes $\zeta_{b}(t)$ for $b \geqslant a_{2}$ ) we get

$$
\zeta_{2}(t) \leqslant a_{2}+2 \sqrt{N t} \text { for } t \geqslant 0
$$

which completes the proof of the theorem.

Now consider the function $v_{0}(x) \equiv A \chi_{\left[-x_{0} x_{0}\right]}(x)$ and let $u_{0} \equiv \psi^{-1} v_{0}$. Although $u_{0}(x)$ is discontinuous, there still exists a weak solution $u(x, t)$ of (1.6), (1.7) in the sense of Sabinina [17]; that is, $u \in L^{2}(G) \cap L^{\infty}(G), u \geqslant 0$ a.e. in $R^{2}$, and $\phi(u)$ has a weak derivative $\partial \phi(u) / \partial x \in L^{2}(G)$ such that (1.9) holds for all $f(x, t) \in C_{0}^{1}(t<T)$. Given $v_{0}(x)$ as above, $u(x, t)$ can be obtained as follows. We define a sequence of Lipschitz functions $\left\{v_{0}^{n}\right\}$ by

$$
v_{0}^{n}(x)= \begin{cases}0 & \text { if }|x| \geqslant x_{0}+1 / n, \\ A & \text { if }|x| \leqslant x_{0}, \\ \text { linear } & \text { otherwise. }\end{cases}
$$

Let $u_{0}^{n} \equiv \psi^{-1} v_{0}^{n}$. Then for each $n \geqslant 1$ we have $\phi\left(u_{0}^{n}\right) \in C^{0,1}\left(R^{1}\right)$ and by Theorem 3.1 there exists a unique weak solution $u^{n}(x, t)$ of (1.6), (1.7) with initial data $u_{0}^{n}(x)$. Let $v^{n} \equiv \psi u^{n}$. By Theorem 4.1 the sequence of functions $\left\{u^{n}\right\}$ is monotone decreasing and has a limit $u(x, t)=\lim _{n \rightarrow \infty} u^{n}(x, t)$ in $G$. Theorem 4.4 implies that $v=\psi(u)$ is Lipschitz continuous in $x$, uniformly in $\{\tau \leqslant t<T\}$ for any $\tau>0$ and in any bounded rectangle $(\xi, \eta) \times[0, T]$ as long as $\underline{\xi}, \eta \notin\left[-x_{0}, x_{0}\right]$. As in the proof of Theorem 3.1, this implies that $u \in C\left(\bar{G} \backslash\left\{\left(-x_{0}, 0\right) \cup\left(x_{0}, 0\right)\right\}\right)$. Also, as in the proof of Theorem 3.1, $u \in C_{2+\alpha}(P[u])$. The existence of an interface follows just as in Theorem 6.1, that is, $P[u]=\left\{(x, t) \in G \mid \zeta_{1}(t)<x<\zeta_{2}(t), 0 \leqslant t \leqslant T\right\}$, where $\zeta_{i} \in$ $\left.C^{0,1}(\tau, T) \cap C[0, t], \zeta_{1}(0)=-x_{0}, \zeta_{2}(0)=x_{0}, \zeta_{1}\right\rangle$ and $\zeta_{2} \succ$. Furthermore, a weak derivative $\partial \phi(u) / \partial x \in L^{2}(G)$ exists such that (1.9) holds. To show this, it suffices to show $\left\{\partial \phi\left(u_{n}\right) / \partial x\right\}$ is bounded in $L^{2}(G)$ and use the fact that 
(1.9) holds with $u$ replaced by $u^{n}$; and this can be shown by considering the equation $\partial u^{n} / \partial t=\partial^{2} \phi\left(u^{n}\right) / \partial x^{2}$ in $P\left[u^{n}\right]$, multiplying by $u$ and integrating by parts over $P\left[u^{n}\right]$ (see $\left.[17, \S 3]\right)$. One then finds that $\left\|\partial \phi\left(u^{n}\right) / \partial x\right\|_{L^{2}(G)}$ is bounded by a constant depending only on sup $u^{n}$ and $\left\|u_{0}^{n}(x)\right\|_{L^{2}\left(R^{1}\right)}$. We then have the following theorem.

THEOREM 8.5. Let $u$ be $a$ weak solution of the Cauchy problem (1.6), (1.7) with initial data $u_{0}(x)$ given by $u_{0} \equiv \psi^{-1} v_{0}$ where $v_{0}(x)=A \chi_{\left[-x_{0}, x_{0}\right]}(x)$ for some constants $A>0, x_{0}$. Let $B=\sup \left\{\sigma^{\prime}(s) \mid 0<s<1+\sup v\right\}$, so that (8.1) holds, and let the functions $u^{n} \searrow u$ be as above. Then,

$$
\alpha \sqrt{t} \leqslant \zeta_{2}(t)-x_{0} \leqslant \beta \sqrt{t}, \quad-\beta \sqrt{t} \leqslant \zeta_{1}(t)+x_{0} \leqslant-\alpha \sqrt{t}
$$

for $0 \leqslant t \leqslant \tau_{0}$ where $\alpha, \beta$ and $\tau_{0}$ are positive constants depending only on $B$ and $A$.

Proof. Let $u_{0}^{n}, v_{0}^{n}, u_{0}, v_{0}, u^{n}, u$ and $v$ be as in the discussion preceding the statement of this theorem. We define a sequence of functions $\left\{\bar{v}_{0}^{n}(x)\right\}$ by

$$
\bar{v}_{0}^{n}(x)= \begin{cases}0 & \text { if }|x| \geqslant x_{0} \\ A & \text { if }|x| \leqslant x_{0}-1 / n, \\ \text { linear } & \text { otherwise, }\end{cases}
$$

and let $\bar{u}^{n}(x, t)$ be the unique weak solution of (1.6), (1.7) with initial data $\bar{u}_{0}^{n}(x) \equiv \psi^{-1} \bar{v}_{0}^{n}(x)$. Clearly, $\bar{v}_{0}^{n} \nearrow$ and $\bar{u}_{0}^{n} \leqslant u_{0}^{n}$. Hence, by Theorem 4.1, $\bar{u}^{n} \leqslant u^{n}$ in $G$ and, consequently, since $u^{n} \succ u$ and $\bar{u}^{n} \nearrow$ we have

$$
\bar{u}^{n}(x, t) \leqslant u(x, t) \leqslant u^{n}(x, t) \text { in } G .
$$

Letting $\bar{\zeta}_{i}^{n}(t), \zeta_{i}(t)$, and $\zeta_{i}^{n}(t)(i=1,2)$ denote the interface curves of $\bar{u}^{n}, u$, and $u^{n}$, respectively, (8.40) implies

$$
\bar{\zeta}_{2}^{n}(t) \leqslant \zeta_{2}(t) \leqslant \zeta_{2}^{n}(t) \text { for } 0 \leqslant t \leqslant T .
$$

Applying Theorem 8.4. to $u^{n}$ we get

$$
\zeta_{2}^{n}(t) \leqslant x_{0}+1 / n+2 \sqrt{A t} \text { for } t \geqslant 0,
$$

and applying Theorem 8.3 to $\bar{u}^{n}$ we get

$$
\bar{\zeta}_{2}^{n}(t) \geqslant x_{0}-1 / n+\theta_{1} \sqrt{A} \sqrt{t} \text { for } 0 \leqslant t \leqslant \tau_{0},
$$

where $\theta_{1}, \tau>0$ depend only on $B$. Equations (8.41)-(8.43) together imply

$$
x_{0}-1 / n+\theta_{1} \sqrt{A} \sqrt{t} \leqslant \zeta_{2}(t) \leqslant x_{0}+1 / n+2 \sqrt{A} \sqrt{t}
$$

for $0 \leqslant t \leqslant \tau_{0}$. Letting $n \rightarrow \infty$ in (8.44) yields

$$
\theta_{1} \sqrt{A} \sqrt{t} \leqslant \zeta_{2}(t)-x_{0} \leqslant 2 \sqrt{A} \sqrt{t} \quad(0 \leqslant t \leqslant \tau) .
$$

The result for $\zeta_{1}(t)$ follows similarly. 
IX. The behavior of the interface curves as $t \rightarrow \infty$. Throughout this section it is assumed that $u(x, t)$ is a weak solution of (1.6), (1.7) with $\phi \in \Phi_{0}$. The initial data $u_{0}(x)$ are assumed to satisfy $(1.8)$ and, as usual, $N=\sup v_{0}$. Also we assume $a_{1}<0$ and $a_{2}>0$.

THEOREM 9.1. For all $t \geqslant 0$ we have

$$
\left|\zeta_{i}(t)\right| \geqslant x_{0}(1+\mu t)^{1 /(B+2)} \quad(i=1,2)
$$

where $B=\sup \left\{\sigma^{\prime}(s) \mid 0<s<\sup v_{0}\right\}$, where $x_{0}>0$ depends on the shape of $v_{0}$, and $\mu=\mu\left(x_{0}, B\right.$, sup $\left.v_{0}\right)>0$.

Proof. Let $B_{\varepsilon}=\sup \left\{\sigma^{\prime}(x) \mid 0<x<\varepsilon+N\right\}$ where $0<\varepsilon<1$. We may assume $v_{0}(0)=N$ and choose $x_{0}>0$ such that $v_{0}(x)>N / 2$ when $|x|<x_{0}$. Then, repeating the argument of Theorem 8.3 leading to equation (8.32), we get

$$
\zeta_{2}(t)>x_{0}+f\left(C_{\varepsilon} / x_{0}, t\right) \text { for } t>0
$$

where $C_{\varepsilon}=\left|2\left(B_{\varepsilon}+1\right)\left(B_{\varepsilon}+2\right) / B_{\varepsilon}\right|^{1 /\left(B_{\varepsilon}+2\right)}$ and $f$ is as defined in the proof of Theorem 8.3. Explicitly, we have

$$
\zeta_{2}(t) \geqslant x_{0}\left(1+\mu_{e} t\right)^{1 /\left(B_{e}+2\right)} \text { for } t \geqslant 0,
$$

where $\mu_{\varepsilon}=N\left(2+B_{\varepsilon}\right) / x_{0}^{2}$. Clearly as $\varepsilon \rightarrow 0, B_{\varepsilon} \rightarrow B$ and $\mu_{\varepsilon} \rightarrow N(2+B) / x_{0}^{2}$ $\equiv \mu$. The result for $\zeta_{1}$ follows similarly.

Theorem 8.4 furnishes the estimates $\left|\zeta_{i}(t)\right| \leqslant\left|a_{i}\right|+2 \sqrt{N t}(i=1,2)$ for all $t>0$, where $N=\sup v_{0}$. The next theorem improves this estimate for large $t$ in the case $\phi(u)=u^{m}(m>1)$.

THEOREM 9.2. Let the assumptions of Theorem 9.1 hold and also assume that for some $m>1$,

$$
\phi(u)=u^{m}
$$

Then

$$
\left|\zeta_{i}(t)\right|<\left(1+\left|a_{i}\right|\right)(\gamma t+1)^{1 /(m+1)}
$$

for all $t>0(i=1,2)$, where $\gamma>0$ depends only on $m, a_{1}, a_{2}$, and $N=$ $\sup v_{0}$.

Proof. Recall the functions $\beta_{K, L}(x, t)$ introduced in the proof of Theorem 8.3. By translation we may assume without loss of generality that $a_{1}=-a_{2}$. It is easy to see that for properly chosen positive constants $K^{\prime}$ and $L^{\prime}$ (depending on $a_{2}, B$, and $N$ ) we have

$$
\begin{aligned}
& \beta_{K^{\prime}, L^{\prime}}(x, 0)=0 \text { iff }|x| \geqslant a_{2}+1, \\
& \beta_{K^{\prime}, L^{\prime}}(x, 0)>v_{0}(x) \text { for } x \in R^{1} .
\end{aligned}
$$


The result then follows by Theorem 4.1 since $\psi^{-1} \beta_{K^{\prime}, L^{\prime}}$ is a weak solution of (1.6), (1.7).

Noting that $\sigma^{\prime} \equiv m-1$ when $\phi(u)=u^{m}(m>1)$, the following corollary is immediate.

COROLlary 9.3. If the assumptions of Theorem 9.2 hold, then there exist positive constants $\alpha$ and $\beta$, depending only on $a_{1}, a_{2}, m$, sup $v_{0}$ and the shape of $v_{0}$ such that

$$
\alpha t^{1 /(m+1)} \leqslant\left|\zeta_{i}(t)\right| \leqslant \beta t^{1 /(m+1)}
$$

when $t>1(i=1,2)$.

Proof. We clearly have, from Theorems 9.1 and 9.2,

$$
x_{0} \mu^{1 /(m+1)} t^{1 /(m+1)}<\left|\zeta_{i}(t)\right|<\left(1+\left|a_{i}\right|\right)(1+\gamma)^{1 /(m+1)} t^{1 /(m+1)}
$$

when $t>1$, and this is the desired result.

$X$. When the interface is strictly monotone.

THEOREM 10.1. Let the hypotheses of Lemma 7.1 be in effect and suppose that $\zeta_{i}^{\prime}(t) \equiv 0$ for $t_{0}<t<t_{1}$ where $t_{0}, t_{1}$ and $i$ are constants such that $0<t_{0}<t_{1}$ and $i=1$ or 2 . Then $\zeta_{i}^{\prime}(t) \equiv 0$ for $0 \leqslant t \leqslant t_{1}$.

Proof. It suffices to consider the case $i=2$. Let $\zeta(t)$ denote $\zeta_{2}(t)$ and suppose that

$$
\alpha \equiv-v_{x}(\zeta(\tau), \tau)>0
$$

for some $\tau \in\left[0, t_{0}\right]$. By Lemma 7.1 there exists a constant $P>0$ such that

$$
v_{x x}(x, t)>-2 P \text { for }(x, t) \in G .
$$

Let a function $\beta_{\tau}(x, t)$ be defined by

$$
\beta_{\tau}(x, t) \equiv\left\{\begin{array}{c}
K\left(\frac{B+1}{B}\right) \lambda^{-B}\left(K L^{2}(t-\tau)\right)\left[1-\frac{L^{2}(x-\nu)^{2}}{\lambda^{2}\left(K L^{2}(t-\tau)\right)}\right] \\
\text { if }|x-\nu| \leqslant L^{-1} \lambda\left(K L^{2}(t-\tau)\right), \\
0 \quad \text { if }|x-\nu| \geqslant L^{-1} \lambda\left(K L^{2}(t-\tau)\right),
\end{array}\right.
$$

where

$$
\begin{gathered}
B \equiv \sigma^{\prime}(0), \quad C \equiv\left[\frac{2(B+1)(B+2)}{B}\right]^{1 /(B+2)}>0, \quad \lambda(t) \equiv C(t+1)^{1 /(B+2)}, \\
L \equiv \frac{2 C P}{\alpha}>0, \quad K \equiv \frac{B}{B+1} \frac{\alpha^{2}}{4 P} C^{B}>0, \quad \nu \equiv \zeta(\tau)-\frac{C}{L}
\end{gathered}
$$


It should be noted that $\beta_{\tau}(x, t)=\beta_{K, L}(x-\nu, t-\tau)$ where $\beta_{K, L}(x, t)$ is defined in the proof of Theorem 8.3. Consequently, $\left(\psi^{-1} \circ \beta_{\tau}\right)(x, t)$ is a weak solution of (1.22) $(m=B+1)$ in $R^{1} \times[\tau, T]$ with initial data $u_{0}(x)=$ $\left(\psi^{-1} \circ \beta_{\tau}\right)(x, \tau)$. Also note that the right interface for $\beta_{\tau}$ is the curve $x=\zeta(t)$ given by

(10.4) $\xi(t) \equiv \zeta(\tau)-\frac{C}{L}+\frac{C}{L}(1+\bar{C} P(t-\tau))^{1 /(B+2)}$ for $t>\tau$, where $\bar{C} \equiv B C^{B+2} /(B+1)=2(B+2)$. Equation (10.2) implies

$$
v(x, \tau)>-\alpha(x-\zeta(\tau))-P(x-\zeta(\tau))^{2}
$$

for $(x, \tau) \in P[u]$.

From (10.3) we get, for $x \in R^{1}$,

$$
\beta_{\tau}(x, \tau)=\max \left[0,-\alpha(x-\zeta(\tau))-P(x-\zeta(\tau))^{2}\right] .
$$

Comparing (10.5) and (10.6) we see that

$$
v(x, \tau)>\beta_{\tau}(x, \tau) \text { for } x \in R^{1} .
$$

An argument similar to the one used in the proof of Theorem 8.3 yields the conclusion

$$
v(x, t)>\beta_{\tau}(x, t) \text { in } R^{1} \times[\tau, T] .
$$

Hence, $\xi\left(t_{1}\right)<\zeta\left(t_{1}\right)=\zeta\left(t_{0}\right)$, which implies that

$$
\zeta(\tau)-(C / L)+(C / L)\left(1+\bar{C} P\left(t_{1}-\tau\right)\right)^{1 /(B+2)}<\zeta\left(t_{0}\right) .
$$

Since $t_{1}-\tau \geqslant t_{1}-t_{0} \equiv \Delta>0$ we get

$$
\zeta(\tau)-(C / L)+(C / L)(1+\bar{C} \Delta)^{1 /(B+2)}<\zeta\left(t_{0}\right) .
$$

Setting $\varepsilon=C\left[(1+\bar{C} \Delta)^{1 /(B+2)}-1\right]>0$, we get

$$
\zeta(\tau)+\varepsilon / L \leqslant \zeta\left(t_{0}\right) .
$$

Recalling that $L=2 C P / \alpha>0$ and setting $\eta=2 C P / \varepsilon>0$, we get

$$
\eta \zeta(\tau)+\alpha \leqslant \eta \zeta\left(t_{0}\right) .
$$

Equation (10.12) was derived assuming $\tau \in\left[0, t_{0}\right]$ and $\alpha \equiv-v_{x}(\zeta(\tau), \tau)>0$. However, (10.12) also holds if $\alpha=0$, since $\zeta$ is monotone increasing. Using the fact (see $\S$ VII) that $\zeta^{\prime}(t)=-v_{x}(\zeta(t), t)$ a.e., we see that $(10.12)$ implies

$$
\eta \zeta(\tau)+\zeta^{\prime}(\tau)<\eta \zeta\left(t_{0}\right) \text { a.e. in }\left[0, t_{0}\right] \text {. }
$$

Multiplying (10.13) by $e^{\eta \tau}$ and integrating from $\tau$ to $t_{0}$ we easily deduce that

$$
\zeta(\tau) \geqslant \zeta\left(t_{0}\right) \text { for any } \tau \in\left[0, t_{0}\right] \text {. }
$$

But by the monotonicity of $\zeta$,

$$
\zeta(\tau)<\zeta\left(t_{0}\right)
$$


and, therefore,

$$
\zeta(\tau)=\zeta\left(t_{0}\right) \text { on }\left[0, t_{0}\right]
$$

This concludes the proof of the theorem.

COROLlaRY 10.2. Under the hypotheses of Theorem 10.1 the right (left) interface is either never vertical or is vertical for some finite duration beginning at $t=0$.

Proof. This follows directly from Theorems 9.1 and 10.1 .

REMARK 10.3. Initially vertical interfaces do exist because of Theorem 8.2.

XI. The gradient of $v$ in $P[u]$. In this section $u(x, t)$ denotes a weak solution of (1.6), (1.7) and $u_{0}(x)$ is assumed to satisfy (1.8). We also assume $\phi \in \Phi_{0}$.

Definition 11.1. Let $I_{\tau}=P[u] \cap\{t=\tau\}$ for $0<\tau<T$. We define $n_{+}(\tau)$ $\left(n_{-}(\tau)\right)$ to be the number, possibly countably infinite, of maximal disjoint open intervals contained in $I_{\tau}$ on which $v_{x}(x, \tau)>0\left(v_{x}(x, \tau)<0\right)$.

THEOREM 11.2. If $v_{0} \equiv \psi\left(u_{0}\right) \in C^{1}(I)$ and if $n_{+}(0)$ and $n_{-}(0)$ are finite, then the functions $n_{+}(t)$ and $n_{-}(t)$ are monotone decreasing for $t \geqslant 0$. Also, there exists a constant $\delta>0$ such that $v_{x}>0$ when $\zeta_{1}(t)<x<\zeta_{1}(t)+\delta, 0<t<$ $T$, and $v_{x}<0$ when $\zeta_{2}(t)-\delta<x<\zeta_{2}(t), 0 \leqslant t \leqslant T$.

PROOF. It suffices to prove the assertions concerning $n_{-}(t)$.

Let $0<\tau<T$. We shall first prove that $n_{-}(\tau)<n_{-}(0)$. We therefore suppose $n_{-}(\tau)>n_{-}(0)$. Let $n=n_{-}(0)$ and let

$$
P_{i}^{0}=\left(c_{i}^{0}, d_{i}^{0}\right) \quad(i=1, \ldots, n)
$$

be the maximal open disjoint intervals on $t=0$ where $v_{0}^{\prime}(x)<0$. We assume they are written left to right, i.e.,

$$
d_{i}^{0}<c_{i+1}^{0} \quad(i=1, \ldots, n-1) .
$$

By the maximality of the intervals $P_{i}^{0}$ it follows that

(11.3) $\quad v_{0}^{\prime}\left(c_{i}\right)=0 \quad(i=1, \ldots, n), \quad v_{0}^{\prime}\left(d_{i}\right)=0 \quad(i=1, \ldots, n-1)$.

Also note that

$$
c_{1}^{0}>a_{1} \text { and } d_{n}^{0}=a_{2} .
$$

Since $n_{-}(\tau)>n_{-}(0)$ there are at least $n+1$ maximal open disjoint intervals $P_{i}^{\tau}$ on $\{t=\tau\}$ on which $v_{x}<0$. Select from this collection a subcollection consisting of precisely $n$ intervals with

$$
P_{i}^{\tau}=\left(c_{i}^{\tau}, d_{i}^{\tau}\right) \quad(i=1, \ldots, n)
$$

and where

$$
d_{i}^{\tau}<c_{i+1}^{\tau} \quad(i=1, \ldots, n-1)
$$


and

$$
d_{n}^{\tau}<\zeta_{2}(\tau)
$$

Again by maximality we have

$$
v_{x}\left(c_{i}^{\tau}, \tau\right)=v_{x}\left(d_{i}^{\tau}, \tau\right)=0 \quad(i=1, \ldots, n) .
$$

Let $A=\left(a_{1}, Q\right)$ be a maximal interval on which $v_{0}^{\prime}>0$. Clearly $Q \leqslant c_{1}^{0}$. Since $v_{0} \in C(I)$ and $v_{0}>0$ on $I$, it is easy to see that there must be a constant $\varepsilon>0$ such that

$$
v_{0}^{-1}\{(0, \varepsilon)\} \subset A \cup P_{n}^{0} .
$$

We shall now show that there exist points $E_{*}=\left(x_{*}, \tau\right)$ and $E^{*}=\left(x^{*}, \tau\right)$ with the following properties:

(i) $\zeta_{1}(\tau)<x_{*}<c_{1}^{\tau}, d_{n}^{\tau}<x^{*}<\zeta_{2}(\tau)$,

(ii) $v\left(x_{*}, \tau\right)=\varepsilon_{*}<\varepsilon, v\left(x^{*}, \tau\right)=\varepsilon^{*}<\varepsilon$,

(iii) $v_{x}\left(x_{*}, \tau\right)>0, v_{x}\left(x^{*}, \tau\right)<0$,

(iv) $\varepsilon_{*}$ and $\varepsilon^{*}$ are noncritical values of $v$.

Indeed (i) and (ii) are automatically satisfied by choosing $E_{*}$ and $E^{*}$ sufficiently close to the endpoints of $I$. Yet, no matter how close we stay to the endpoints of $I$ we must be able to find points satisfying (iii), since otherwise, by the mean value theorem, there would be points in $P[u]$ where $v \leqslant 0$. Having chosen points satisfying (i), (ii), (iii), Sard's Theorem and the continuity of $v$ and $v_{x}$ in $P[u]$ ensure that we may find nearby points satisfying (i)-(iv).

By Lemma 6.4 there exist level curves $x=\beta_{*}(t)$ and $x=\beta^{*}(t)$ connecting $E_{*}$ to $A$ and $E^{*}$ to $P_{0}^{n}$, along which $v \equiv \varepsilon_{*}, v_{x}>0$ and $v \equiv \varepsilon^{*}, v_{x}<0$, respectively. The fact that $\beta_{*}(0) \in A$ and $\beta^{*}(0) \in P_{0}^{n}$ follows by Remark 5.6 and equation (11.8).

As in Remark 5.6 it follows that there are only countably many values $\left\{\eta_{i}\right\}_{i=1}^{\infty}$ such that $v_{0}^{\prime}(x)=\eta_{i}$ on some interval $Z_{i} \subset I$. Let $S$ denote the set of critical values of $v_{x}(x, t)$, i.e. the set of numbers $\alpha$ such that $v_{x x}(x, t)=$ $v_{x t}(x, t)=0$ and $v_{x}(x, t)=\alpha$ at some point $(x, t) \in P[u]$. By Sard's Theorem $S$ has measure zero. If each $i=1, \ldots, n$, choose a point $E_{i}=\left(x_{i}, \tau\right)$ in $P_{i}^{\tau}$ so that $v_{x}\left(x_{i}, \tau\right)=\alpha_{i}<0, \alpha_{i} \notin S \cup\left\{\eta_{i}\right\}, v_{x x}\left(x_{i}, \tau\right) \neq 0$ and all $\alpha_{i}$ are distinct. This is clearly possible because $v_{x}$ and $v_{x x}$ are continuous in $P[u]$. Indeed $v_{x x}(x, \tau) \neq 0$ on $P_{i}^{\tau}$ because of (11.7), and, around any point in $P_{i}^{\tau}$ where $v_{x x} \neq 0$ there must be a neighborhood in which both $v_{x x} \neq 0$ and $v_{x}$ assumes a continuum of values.

According to Lemma 5.7 there exist level curves $x=\beta_{i}(t)$ through $E_{i}$ extending downward along which $v_{x}=\alpha_{i}$ and $v_{x x} \neq 0$. Since all $\alpha_{i}$ are distinct, none of these curves can intersect.

Now suppose $\beta^{*}$ intersects $\beta_{n}$ at some point $\left(x_{1}, t_{1}\right)$ in $P[u] \cup I$. Let $\left(x_{0}, t_{0}\right)$ 
be the first such point at which they intersect, i.e. $0 \leqslant t_{0}<\tau$ and $t_{0}$ is as large as possible. Let $D$ denote the domain bounded above by the line $t=\tau$ and below this line by the curves $x=\beta^{*}(t)$ and $x=\beta_{n}(t)$ for $t_{0}<t<\tau$. The function $v^{(1)}(x, t) \equiv v_{x}(x, t) \in C(\vec{D})$ satisfies the uniformly parabolic equation

$$
v_{t}^{(1)}=\sigma(v) v_{x x}^{(1)}+\left(\sigma^{\prime}(v)+2\right) v_{x} v_{x}^{(1)} \text { in } D .
$$

Since $v^{(1)}<0$ on $\beta_{n}$ and $\beta^{*}$, the maximum principle implies $v^{(1)}<0$ in $\bar{D}$. But $\left(d_{n}^{\tau}, \tau\right) \in \bar{D}$ and $v^{(1)}\left(d_{n}^{\tau}, \tau\right)=0$, a contradiction. Hence $\beta_{n}$ and $\beta^{*} \operatorname{do}$ not intersect.

Clearly $\beta_{*}$ cannot intersect $\beta_{1}$ since the sign of $v_{x}$ is different on each curve. Hence none of the $\beta_{i}(i=1, \ldots, n)$ can exit $P[u]$ at the interface, and therefore, as in Remark 5.6, it follows from our choice of the values $\left\{\alpha_{i}\right\}$ that $\lim _{t \rightarrow 0} \beta_{i}(t)$ exists for $i=1, \ldots, n$. We conclude

$$
\beta_{*}(t)<\beta_{1}(t)<\cdots<\beta_{n}(t) \text { for } 0 \leqslant t \leqslant \tau \text {. }
$$

We shall now show that no two of the values $\beta_{1}(0), \ldots, \beta_{n}(0), \beta^{*}(0)$ can be contained in the same interval $P_{i}^{0}$ for any $1<i<n$. Suppose $\beta_{k}(0), \beta_{k+1}(0) \in P_{i}^{0}$ where $1<k \leqslant n$ and where we define $\beta_{n+1}=\beta^{*}$. Let $D$ be the domain bounded above by $\{t=\tau\}$, below by $P_{i}^{0}$ and laterally by the curves $\beta_{k}$ and $\beta_{k+1}$. The same maximum principle argument given above again applies and furnishes a contradiction.

Thus we have shown that the curves $\beta_{1}, \ldots, \beta_{n+1}$ define a 1-1 correspondence between the sets $\left\{P_{i}^{\tau}\right\}_{i=1}^{n} \cup\left\{E^{*}\right\}$ and $\left\{P_{j}^{0}\right\}_{j=1}^{n}$ which is ridiculous. Hence $n_{-}(\tau) \leqslant n_{-}(0)$.

To prove the first assertion let $0 \leqslant s<\gamma<T$. Since we have already shown $n_{-}(s) \leqslant n_{-}(0)$ and $n_{-}(\gamma)<n_{-}(0)$, it follows that $n_{-}(s)$ and $n_{-}(\gamma)$ are finite; by the argument above we conclude $n_{-}(\gamma) \leqslant n_{-}(s)$.

To prove the last assertion we first construct a level curve $v \equiv \varepsilon_{0}$ given by $x=\beta(t) \in C[0, T]$ where $\varepsilon_{0}>0$ is chosen so small that we are sure $\beta(0)$ is in the rightmost interval where $v_{0}^{\prime}<0$. We constructed such a function above. We shall now show $\beta(t) \in P_{t}^{n_{-}(t)}$ for each $0<t<T$, where $P_{t}^{n_{-}(t)}=$ $\left(C_{n_{-}(t)}^{t}, \zeta_{2}(t)\right)$. If for some $0<\tau<T$ we have $\beta(\tau)<C_{n_{-}(\tau)}^{\tau}$ it most be that $\beta(\tau)<C_{n_{-}(\tau)}^{\tau}$ since $v_{x}$ never vanishes on $\beta$ and since $v_{x}\left(C_{n_{-}(\tau)}^{\tau}, \tau\right)=0$. In this case we produce another level curve $v \equiv \varepsilon_{1}<\varepsilon_{0}$ given by $x=\bar{\beta}(t) \in C[0, \tau]$ such that $\bar{\beta}(\tau)>C_{n_{-}(\tau)}^{\tau}$. Since $\varepsilon_{1} \neq \varepsilon_{0}, \beta$ and $\bar{\beta}$ cannot intersect. Consider the domain $D$ bounded above by $t=\tau$, below by $P_{n}^{0}\left(C_{n}^{0}, a_{2}\right)$ and laterally by $\beta$ and $\bar{\beta}$. On the lower and lateral boundaries we have $v_{x}<0$, and at the point $\left(C_{n_{-}(\tau)}, \tau\right)$ on the upper boundary we have $v_{x}=0$, which, by the maximum principle, is impossible. Thus the second assertion is proved.

Recall that $w \equiv \phi(u), w_{0} \equiv \phi\left(u_{0}\right), v \equiv \psi(u)$ and $v_{0} \equiv \psi\left(u_{0}\right)$. We then have the following theorem. 
THEOREM 11.3. If $v_{0} \in C^{\prime}(\bar{I})$ and $w_{0}^{\prime \prime}>0$ near $a_{1}$ and $a_{2}$ then $v_{1}>0$ in some neighborhood $N_{i}$ of $\zeta_{i}(i=1,2)$, where for some $\delta>0$,

$$
\begin{aligned}
& N_{1}=\left\{(x, t) \mid \zeta_{1}(t)<x<\zeta_{1}(t)+\delta, 0 \leqslant t \leqslant T\right\}, \\
& N_{2}=\left\{(x, t) \mid \zeta_{2}(t)-\delta<x<\zeta_{2}(t), 0 \leqslant t \leqslant T\right\} .
\end{aligned}
$$

Proof. The function $w(x, t)$ satisfies $w_{t}=a(w) w_{x x}$ in $P[u]$ where $a(w)=$ $\phi^{\prime}(\Phi(w))$. Since $w=\left(\phi \circ \psi^{-1}\right)(v)$, we see that the sign of $w_{t}$ agrees with the sign of $v_{t}$ in $P[u]$. In fact, using (1.16), we see that $w_{t}=u v_{t}, w_{x}=u v_{x}$ in $P[u]$. Also, since by Theorem $4.4,\left|v_{x}\right|$ is bounded in $P[u]$, it follows that $w_{x}$ approaches zero when we approach the interface.

We shall now show $w_{t}>0$ near $\zeta_{2}$ (the proof for $\zeta_{1}$ being similar). Suppose $w_{0}^{\prime \prime}>0$ for $a_{2}-\delta_{0}<x<a_{2}$. By Theorem 11.2 we know $w_{x}<0$ in

$$
N_{\delta_{0}}=\left\{(x, t) \mid \zeta_{2}(t)-\delta_{0}<x<\zeta_{2}(t), 0 \leqslant t \leqslant T\right\}
$$

where $\delta_{0}>0$ is chosen smaller if necessary. Using Lemma 5.3 and the techniques of Theorem 11.2 we can produce a family of level curves $w_{x}=\alpha_{i}$ $<0$, where $\alpha_{i} \rightarrow 0$ as $i \rightarrow \infty$, lying in $N_{\delta_{0}}$ given by $x=\xi_{i}(t) \in C[0, T]$ and such that $w_{x x}>0$ on $\xi_{i}$ and such that $\xi_{i}(T) \nearrow \zeta_{2}(T)$ and $\xi_{i}(0) \nearrow \zeta_{2}(0)=a_{2}$. The points $\left(\xi_{i}(T), T\right)$ can be chosen such that $w_{x x}>0$ at these points, since if $w_{x x} \leqslant 0$ near $\left(\zeta_{2}(T), T\right)$ in $P[u]$ then $w<0$ on the line $\{t=T\}$ near $\left(\zeta_{2}(T), T\right)$.

Clearly $\xi_{i}(t) \nearrow \zeta_{2}(t)$ for any $t \in[0, T]$ and, by Dini's theorem, the convergence is uniform on $[0, T]$. For arbitrary $i>1$, denote by $D_{i}$ the domain in $P[u]$ that is bounded below by $\{t=0\}$, above by $\{t=T\}$, and laterally by $\xi_{i}$ and $\xi_{i+1}$. We shall show that $w_{t}>0$ in $D_{i}$. Let $z=w_{t}$ and note that $z$ satisfies the uniformly parabolic equation

$$
L[z] \equiv a(w) z_{x x}-z_{t}=-\left(a^{\prime}(w) / a(w)\right) z^{2}<0
$$

in $D_{i}$. Since $z \in C\left(\overline{D_{i}}\right), z \geq 0$ on $\partial D_{i} \cap\{t=0\}$ and $z>0$ on the lateral boundary of $D_{i}$, the maximum principle implies $z>0$ in $\bar{D}_{i} \backslash \partial D_{i} \cap\{t=0\}$. The assertion of the theorem then follows from the arbitrariness of $i$.

TheOREM 11.4. The set $S \equiv\left\{(x, t) \mid(x, t) \in P[u], v_{x}(x, t)=0\right\}$ has no interior points.

Proof. Suppose $v_{x} \equiv 0$ in some ball $B\left(P_{0}, r\right) \subset P[u]$, where $P_{0}=\left(x_{0}, t_{0}\right)$ and $>0$. Let $R_{n}$ denote the rectangle

$$
\left(x_{0}, \zeta_{2}\left(t_{0}-r /(n+2)\right)\right) \times\left(t_{0}-r /(n+2), t_{0}+r /(n+2)\right) .
$$

Since $\bar{R}_{n} \subset P[u]$, it follows that $\inf _{\bar{R}_{n}} v>0$ and the operator

$$
M \equiv \partial / \partial t-\sigma(v(x, t))\left(\partial^{2} / \partial x^{2}\right)
$$

is uniformly parabolic on $R_{n}$. Let $\alpha=v\left(x_{0}, t_{0}\right)$ and define a function $z$ : 
$R^{2} \rightarrow R^{1}$ by $z=v-\alpha$. Then

$$
M z=v_{t}-\sigma(v) v_{x x}=v_{x}^{2}
$$

in $R_{n}$. Since $z \equiv 0$ and $z_{x} \equiv 0$ on the left side of $R_{n}$, [16, Theorem 4] implies $z \equiv 0$ in $R_{n}$. Letting $n \rightarrow \infty$ we see that $z \equiv 0$, and, consequently, that $v \equiv \alpha$, on the horizontal line segment connecting $\left(x_{0}, t_{0}\right)$ to the right interface. The continuity of $v$ implies $\alpha=0$; but this is impossible, since $v>0$ in $P[u]$.

ACKNOWLEDGEMENT. The author of this paper would like to express his deep appreciation to his dissertation advisor, Professor Avner Friedman, for suggesting this research topic and many of the problems solved in this paper, and for his many valuable criticisms.

\section{REFERENCES}

1. D. G. Aronson, Regularity properties of flows through porous media: The interface, Arch. Rational Mech. Anal. 37 (1970), 1-10. MR 41 \#656.

2. Regularity properties of flows through porous media: A counterexample, SIAM J. Appl. Math. 19 (1970), 299-307. MR 42 \#683.

3. $\longrightarrow$ Regularity properties of flows through porous media, SIAM J. Appl Math. 17 (1969), 461-467. MR 40 \#571.

4. G. I. Barenblatt, On one class of exact solutions of the plane one-dimensional problem of unsteady filtration of a gas in a porous medium, Akad. Nauk SSSR Prikl. Mat. Meh. 17 (1953), 739-742. MR 16, 298.

5. $\quad$ On some unsteady motions of a liquid and a gas in a porous medium, Akad. Nauk SSSR Prikl. Mat. Meh. 16 (1952), 67-78. MR 13, 700.

6. A. Friedman, Partial differential equations of parabolic type, Prentice-Hall, Englewood Cliffs, N.J., 1964. MR 31 \#6062.

7. A. S. Kalašnikov, On the differential properties of generalized solutions of equations of the nonsteady filtration type, Vestnik Moskov. Univ. Ser. I Mat. Meh. 29 (1974), 62-68 = Moscow Univ. Math. Bull. 29 (1974), 48-53. MR 49 \# 7578.

8. On equations of the nonstationary filtration type in which the perturbation is propagated at infinite velocity, Vestnik. Moscov. Univ. Ser. I Mat. Meh. 27 (1972), $45-49$ = Moscow Univ. Math. Bull. 27 (1972), 104-108 (1973). MR 49 \#849.

9. Formation of singularities in solutions of the equation of nonstationary filtration, $\dot{Z}$. Vyčisl. Mat. i Mat. Fiz. 7 (1967), 440-444. MR 35 \# 1940.

10. S. Kamenomostskaya, The asymptotic behavior of the solution of the filtration equation, Israel J. Math. 14 (1973), 76-87. MR 47 \#3841.

11. O. A. Ladyženskaja, V. A. Solonnikov and N. N. Ural'ceva, Linear and quasilinear equations of parabolic type, "Nauka", Moscow, 1967; English transl., Transl. Math. Monographs, vol. 23, Amer. Math. Soc., Providence, R. I., 1968. MR 39 \#3159 a,b.

12. M. Muskat, The flow of homogeneous fluids through porous media, McGraw-Hill, New York, 1937.

13. O. A. Oleinik, On some degenerate quasilinear parabolic equations, Seminari 1962/63 Anal. Alg. Geom. e Topol., Vol. 1, Ist.Naz. Alta Mat. Ediz. Cremonese, Rome, 1965, pp. 355-371. MR 33 \#432.

14. O. A. Oleìnik, A. S. Kalašnikov and Yui-Lin' Čžou, The Cauchy problem and boundary problems for equations of the type of non-stationary filtration, Izv. Akad. Nauk SSSR Ser. Mat. 22 (1958), 667-704. (Russian) MR 20 \#6271.

15. R. E. Prattle, Diffusion from an instantaneous point source with a concentration-dependent coefficient, Quart. J. Mech. Appl. Math. 12 (1959), 407-409. 
16. M. H. Protter, Properties of solutions of parabolic equations and inequalities, Canad. J. Math. 13 (1961), 331-345. MR 27 \#3943.

17. E. S. Sabinina, On the Cauchy problem for the equation of nonstationary gas filtration in several space variables, Dokl. Akad. Nauk SSSR 136 (1961), 1034-1037 = Soviet Math. Dokl. 2 (1961), 166-169. MR 28 \#1416.

Department of Mathematics, University of Kentucky, Lexington, Kentucky 40506 